\title{
Distribución de macroinvertebrados (Plecoptera y Aeglidae) en ecosistemas fluviales de la Patagonia chilena: ¿Muestran señales biológicas de la evolución geomorfológica postglacial?
}

\author{
Distribution of macroinvertebrates (Plecoptera and Aeglidae) in fluvial ecosystems of the \\ Chilean Patagonia: Do they show biological signals of the postglacial geomorphological \\ evolution?

\section{CLAUDIO VALDOVINOS $1,2,{ }^{*}$, ANDREA KIESSLING ${ }^{1}$, MARÍA MARDONES ${ }^{3}$, CAROLINA MOYA $^{1}$, ALEJANDRA OYANEDEL ${ }^{1}$, JACQUELINE SALVO $^{1}$, VIVIANA OLMOS ${ }^{1} \&$ ÓSCAR PARRA $^{1,2}$}

\footnotetext{
${ }^{1}$ Centro de Ciencias Ambientales EULA, Universidad de Concepción, Casilla 160-C, Concepción, Chile

${ }^{2}$ Centro de Investigación de Ecosistemas de la Patagonia (CIEP), Coyhaique, Chile

${ }^{3}$ Departamento de Ciencias de la Tierra, Universidad de Concepción, Casilla 160-C, Concepción, Chile

* Autor correspondiente: cvaldovi@udec.cl
}

\begin{abstract}
RESUMEN
La Patagonia chilena incluye uno de los sistemas hídricos más complejos de Sudamérica y uno de los menos intervenidos del mundo, los cuales fueron intensamente modelados por la actividad glacial del Cuaternario. El objetivo de este estudio fue determinar si en esta área existen zonas de endemismo de macroinvertebrados bentónicos fluviales y analizar los patrones de diversidad de organismos de alta (Plecoptera) y baja vagilidad (Aeglidae). Entre el 2006 y 2009 se muestrearon 183 sitios entre $42^{\circ} 50^{\prime}$ y $54^{\circ} 43^{\prime} \mathrm{S}$, focalizándose en las principales cuencas. Los muestreos se realizaron con una red "kicknet", cubriendo una superficie de fondo de $8 \mathrm{~m}^{2}$. En toda el área se registraron 30 especies de Plecoptera y dos de Aeglidae. Se identificó una zona de endemismo en la cuenca del río Aysén, sustentada por el plecóptero Ceratoperla fazi y el cangrejo Aegla neuquensis. Esta última especie correspondería a una población relictual procedente de los ríos de la estepa Argentina, que habría quedado atrapada en la Patagonia chilena, luego que se invirtiera el flujo del cauce del río que originalmente fluía hacia el Atlántico. Un marcado cambio latitudinal en la diversidad de especies fue evidente a lo largo del área de estudio $\left(\mathrm{R}^{2}\right.$ $=0.72, \mathrm{P}<0.05)$, con una disminución de la riqueza hacia el sur. La regresión entre riqueza de especies $\mathrm{y}$ temperatura media anual del aire fue estadísticamente significativa $\left(\mathrm{R}^{2}=0.67, \mathrm{P}<0.05\right)$, sugiriendo que la hipótesis especies-energía asociada al efecto de las entradas de energía solar, puede tener un efecto relevante sobre la diversidad. Se sugiere que en los organismos de alta vagilidad los acontecimientos históricos tendrían menos influencia que las exigencias ecológicas actuales. Por el contrario, en el caso de los de baja vagilidad, los fenómenos históricos parecieran tener mayor relevancia.
\end{abstract}

Palabras clave: Aegla, biodiversidad, biogeografía, Patagonia, Plecoptera.

\section{ABSTRACT}

The Chilean Patagonia includes one of the most complex hydrological systems of South America and one of the least modified systems of the world. These systems were intensely modified by the glacial activity of the Quaternary. The objectives of this study were to determine whether or not in this area exists zones of endemic benthic fluvial macroinvertebrates, and to analyze the patterns of diversity of species composed by organisms of high (Plecoptera) and low (Aeglidae) vagility. A collection of 183 selected sites, located between the latitude $42^{\circ} 50^{\prime}$ and $54^{\circ} 43^{\prime} \mathrm{S}$, were sampled during 2006 and 2009. The sampling was focused on the main river basins and a "kicknet" was used to cover a surface of $8 \mathrm{~m}^{2}$ at the bottom of the river channel. In all sampled areas, thirty Plecoptera and two Aeglidae species were recorded. One zone dominated by endemic species was identified in the Aysen river basin, which included the Plecoptera Ceratoperla fazi and the crab Aegla neuquensis. The last species belong to an ancient population found in the river steppes of Argentina, and may have been locked in the Chilean Patagonia when the channel flow was reversed from its original discharge towards the Atlantic Ocean. A distinct latitudinal change in the diversity of species was noticed throughout the study area $\left(\mathrm{R}^{2}=0.72, \mathrm{P}<0.05\right)$, showing a gradient of decreasing species richness towards the south. The regression analysis between species richness and annual mean temperature was significant $\left(\mathrm{R}^{2}=0.67, \mathrm{P}<0.05\right)$. This suggests that the input of solar energy had an important effect on species diversity. In addition, the historical events would have had weaker impacts relative to current ecological requirements on organisms of high vagility. Conversely, low vagility organisms seemed to be largely affected by history.

Key words: Aegla, biodiversity, biogeography, Patagonia, Plecoptera. 


\section{INTRODUCCIÓN}

La Patagonia chilena incluye uno de los sistemas hídricos más complejos de Sudamérica y uno de los menos intervenidos del mundo (Carrasco et al. 2002), caracterizada por poseer un frágil y valioso patrimonio ambiental constituido por una gran variedad de ecosistemas lóticos, lénticos y estuarinos (ver Niemeyer \& Cereceda 1984, Valdovinos 2004). En este territorio existen cuatro grandes cuencas hidrográficas con ríos caudalosos trasandinos de régimen mixto (Palena, Cisnes, Aysén y Baker), dos cuencas con alimentación glacial (Pascua y Bravo) y dos extensos campos de hielo (Campo de Hielo Norte y Campo de Hielo Sur). Se estima que dentro de las grandes cuencas de esta región aún se encuentran algunos de los pocos lugares de la Tierra donde no hay efectos significativos de las actividades antrópicas sobre su biodiversidad (Mittermeier et al. 2003, Moya et al. 2009). En estos ecosistemas dulceacuícolas todavía quedan vacíos de conocimiento. Sin embargo, en el caso de macroinvertebrados bentónicos fluviales, con los antecedentes existentes, se ha podido constatar la presencia de una importante biodiversidad de especies, áreas de endemismo y patrones biogeográficos particulares (Illies 1969, Ringuelet 1961, Valdovinos 2006). Sus estructuras habrían sido fundamentalmente el resultado de los acontecimientos históricos del Cuaternario combinados con las exigencias ecológicas actuales de las especies.

Existen evidencias de que cambios ambientales asociados con los ciclos glaciales del Cuaternario han alterado la distribución y estructura genética de la flora y fauna de Norteamérica y Europa (Hewitt 2000, Hausdorf \& Henning 2003). Sin embargo, existen escasos antecedentes sobre estos efectos en el hemisferio Sur (Ruzzante et al. 2008). En Sudamérica, la extensión de las glaciaciones variaron significativamente durante el Cuaternario, y hay evidencias de que los glaciares cambiaron los patrones de drenaje, la distribución de los lagos e incluso la posición de la divisoria continental (Glasser et al. 2008). Los glaciares patagónicos estuvieron restringidos principalmente a la Cordillera Principal a lo largo de gran parte de su rango latitudinal, permaneciendo libres de hielo, la costa de Chile hacia el oeste y parte de la estepa Argentina hacia el este (Ruzzante et al. 2008).

Los efectos de los repetidos avances y retrocesos glaciales sobre la flora y fauna patagónica son prácticamente desconocidos. Diferentes taxa probablemente tengan respuestas diferentes. Por ejemplo, mientras algunas plantas terrestres parecen haber sobrevivido al último ciclo glacial en pequeños refugios australes al oeste de los Andes (Muellner et al. 2005), las poblaciones de roedores actuales han sido el resultado de recolonización de zonas más al norte no glaciadas (Palma et al. 2002). Todavía menos conocidos son los efectos de los ciclos glaciales del Cuaternario sobre la fauna dulceacuícola patagónica. Una excepción corresponde al efecto de estos ciclos sobre la dinámica de algunas poblaciones ancestrales de peces (i.e., Galaxias platei Steindachner, 1898, Percichthys trucha Cuvier \& Valenciennes, 1833), inferida a partir de estudios filogeográficos (Ruzzante et al. 2008). En el caso de los invertebrados bentónicos, se desconocen sus efectos sobre los patrones espaciales de diversidad, o las rutas de recolonización de las zonas deglaciadas, entre otros aspectos. Oyanedel et al. (2008) realizaron un análisis de los patrones de distribución de la biota pelágica dulceacuícola de las cuencas de Chile (i.e., fitoplancton, zooplancton y peces), sugiriendo que en la actualidad la latitud ejerce una fuerte influencia en la distribución de especies. Además, destacan la importancia del grado de vagilidad de los grupos y el grado de estructuración de la metacomunidad, relacionada con el número de áreas de endemismos. Un patrón biogeográfico anidado también fue revelado para un subconjunto de la base de datos anterior (Ramos-Jiliberto et al. 2009).

En este estudio se analizaron, a lo largo de ca. $1,400 \mathrm{~km}$ de la Patagonia chilena, los patrones actuales de distribución espacial de dos tipos de macroinvertebrados bentónicos que difieren marcadamente en sus capacidades de dispersión: a) especies de alta vagilidad, caracterizados por poseer estados adultos alados y por lo tanto una elevada capacidad de desplazamiento, y b) especies de baja 
vagilidad, que no poseen estados adultos alados. Ello se realiza con el objeto de determinar si los patrones de diversidad y endemismo de estos grupos contrastantes evidencian señales biológicas de la evolución geomorfológica fluvial postglacial en esta área de la Patagonia.

Dentro de los diversos grupos taxonómicos que habitan los ecosistemas fluviales de la Patagonia, se seleccionaron los Plecoptera como representantes de organismos de alta vagilidad (Sheldon 1984), y a crustáceos malacostracos de la familia Aeglidae Dana, 1852, como organismos de baja vagilidad (Jara et al. 2006). Estos grupos de organismos fueron elegidos debido al adecuado conocimiento taxonómico disponible, lo que permite la identificación de la mayoría de los taxa a nivel de especie, además de disponer de información sobre sus áreas de distribución geográfica y morfometría de los estados adultos, para la mayoría de las especies.

Uno de los principales componentes de la fauna de invertebrados de ecosistemas fluviales patagónicos corresponde a estados ninfales de Plecoptera pertenecientes al suborden Arctoperlaria Zwick, 1969 (Fochetti \& Tierno de Figueroa 2008). De acuerdo a Vera \& Camousseight (2006), en Chile se encuentran representados por 63 especies pertenecientes a seis Familias: Eustheniidae Tillyard, 1921 (géneros Neuroperlopsis y Neuroperla), Diamphipnoidae Ricker, 1950 (Diamphipnoa y Diamphipnopsis), Austroperlidae Tillyars, 1921 (Andesobius, Klapopteryx y Penturoperla), Gripopterygidae Enderlein, 1909 (Andiperla, Andiperlodes, Antarctoperla, Araucanioperla, Aubertoperla, Ceratoperla, Chilenoperla, Claudioperla, Limnoperla, Megandiperla, Notoperla, Notoperlopsis, Pelurgoperla, Plegoperla, Potamoperla, Rhitroperla, Senzilloides y Teutoperla), Notonemouridae Ricker, 1950 (Austronemoura, Neofulla, Neonemoura y Udamocercia) y Perlidae Latreille, 1802 (Inconeuria, Kempnyella, Nigroperla y Pictetoperla).

Respecto de la familia Aeglidae, incluye a decápodos dulceacuícolas endémicos de la región Neotropical de Sudamérica. Ellos pertenecen a un solo género, Aegla, que reúne a ca. 70 especies y subespecies distribuidas en Chile, Brasil, Argentina, Uruguay y Bolivia.
Chile posee 19 especies y subespecies de Aegla, incluyendo 16 taxa endémicos (BondBuckup \& Buckup 1994, Pérez-Losada et al. 2002a, 2002b). La mayor diversidad de especies ha sido descrita para al territorio chileno comprendido entre el río Maule y Chiloé (Jara et al. 2006). Para la Patagonia chilena se han registrado dos especies: a) Aegla neuquensis Schmitt, 1942, especie ampliamente distribuida en el sector patagónico argentino (e.g., Provincias de Neuquén, Río Negro y Chubut), pero que también ha sido reportada en Chile en la cuenca del río Aysén (Jara et al. 2006), y b) Aegla alacalufi Jara \& López, 1981, que se distribuye ampliamente en Chile desde Chiloé hasta la isla Madre de Dios (Jara et al. 2006).

E1 conocimiento detallado de la distribución de los patrones espaciales de diversidad biológica permite su interpretación biogeográfica identificando áreas naturales que concentren por una parte la mayor cantidad de taxa endémicos, y por otra, que estos endemismos reflejen la historia evolutiva de la diversidad y su relación con biotas ancestrales. Los análisis biogeográficos pueden ser complementados con índices de diversidad filogenética y de riqueza de especies presentes en dichas áreas (Eguiarte et al. 1999, Camus 2001). Entre los métodos utilizados por la biogeografía histórica, el análisis de parsimonia de endemismos $($ Parsimony Analysis of Endemicity = PAE) (Morrone 1994, Escalante \& Morrone 2003, Oyanedel et al. 2008) permite clasificar áreas o localidades de acuerdo a taxa compartidos (Morrone \& Crisci 1995) y, al mismo tiempo, detectar áreas con concentración de especies endémicas (Posadas 1996, Posadas \& MirandaEsquivel 1999, Cavieres et al. 2001, 2002). Los taxa endémicos son aquellos que están delimitados a algún lugar geográfico específico y pueden considerarse como la diversidad biológica única de un lugar (Cavieres et al. 2001). Por el hecho de tener un rango de distribución restringido, los taxa endémicos tienen mayores probabilidades de extinguirse (Myers et al. 2000), por lo cual la delimitación de áreas de endemismo es fundamental para formular estrategias dirigidas al uso sustentable y la conservación de la biodiversidad (Szumik et al. 2002). En este contexto, PAE corresponde a una poderosa 
herramienta biogeográfica para la identificación de "hotspots" de biodiversidad (Garrafoni et al. 2006). Esta metodología ha sido usada recientemente por Oyanedel et al. (2008) en el estudio de los patrones de distribución de la biota pelágica dulceacuícola chilena.

Dada la heterogeneidad de ambientes característicos de la región patagónica chilena (Di Castri 1968), cabría esperar que los diferentes taxa estudiados mostraran patrones distribucionales concordantes con las diferentes zonas descritas en el área y que las discontinuidades distribucionales presentadas por los taxa se reflejaran en áreas de endemismo. Los objetivos del presente estudio fueron, por lo tanto, determinar áreas de endemismo para taxa de elevada y baja vagilidad mediante un análisis de parsimonia de endemismo, analizar los posibles factores causales de tales patrones empleando análisis de los patrones latitudinales de diversidad y de anidados, e interpretar las relaciones biogeográficas que puedan existir entre las distintas cuencas hidrográficas, en relación al efecto de las glaciaciones plesitocénicas que afectaron el sur de Chile.

\section{MÉTODOS}

\section{Área de estudio}

La composición taxonómica y distribución geográfica de los Plecoptera se determinó a partir de muestreos realizados en el período estival de los años 2006 a 2009. El estudio cubrió toda el área de la Patagonia chilena (ca. $1,400 \mathrm{~km})$, localizada entre Chaitén $\left(42^{\circ} 50^{\prime} \mathrm{S}\right)$ y el Parque Nacional Alberto D’Agostini (PNAA, 54 ${ }^{\circ} 43^{\prime} \mathrm{S}$ ) (Fig. 1). En este territorio, el muestreo se focalizó en las cuencas hidrográficas de mayor superficie territorial (i.e. río Yelcho $\mathrm{n}=18$, río Palena $\mathrm{n}=29$, río Cisnes $\mathrm{n}=22$, río Aysén $\mathrm{n}=50$, río Baker $\mathrm{n}=52$, río Pascua $\mathrm{n}=3$, y cuencas del Parque Nacional Alberto D'Agostini /PNAA $\mathrm{n}=9$ ), incluyendo un total de 183 sitios (los datos de composición y riqueza de especies de los sitios localizados en PNAA, se obtuvieron de Moorman et al. 2006). Todos los sitios de muestreo fueron georreferenciados mediante un GPS Garmin GPSMAP 60CSX.

\section{Composición y diversidad de especies}

Con el objeto de estandarizar la tipología de hábitats fluviales estudiados, los muestreos de macroinvertebrados bentónicos se realizaron en arroyos de aguas corrientes (0.1-0.6 m s $\left.\mathrm{m} \mathrm{s}^{-1}\right)$, de escasa profundidad $(0.1-0.5 \mathrm{~m})$ y con fondos de bolones (50$150 \mathrm{~mm}$ de diámetro medio). Estos se realizaron empleando una red tipo "kicknet" de $250 \mu \mathrm{m}$ de abertura de trama (Hauer \& Resh 2007), cubriendo una superficie total de fondo de $8 \mathrm{~m}^{2}$ en un período de muestreo de 20 minutos. Las muestras biológicas fueron preservadas en etanol al $75 \%$, y luego trasladadas al laboratorio para la posterior separación e identificación hasta el nivel taxonómico más bajo posible, empleando un estereomicroscopio. La identificación de los Plecoptera se realizó siguiendo a Caamaño (1985), Heckman (2003), McLellan et al. (2005), McLellan \& Zwick (2007) y Stark (2007, 2008), y a través de la comparación con material depositado en el Museo de Zoología de la Universidad de Concepción (MZUC).

\section{Análisis de parsimonia de endemismos}

Para la identificación de áreas de endemismo se realizó un Análisis de Parsimonia de Endemismos (PAE) de las cuencas hidrográficas estudiadas, las cuales se comportan como "islas" para el caso de la fauna dulceacuícola, al estar limitadas por la línea divisoria de aguas en su área continental y por las aguas marinas costeras en su zona terminal (Fig. 1). Este análisis se realizó siguiendo la metodología utilizada por Morrone (1994). Se empleó análisis de parsimonia de caracteres ordenados (Parsimonia de Wagner), para lo cual se elaboró una matriz, donde las columnas representan las cuencas y las filas las especies; se agregó una fila hipotética con ausencias para enraizar los cladogramas, y se registró la ausencia o presencia $(0,1)$ de cada especie en las diferentes cuencas estudiadas. El análisis de la matriz se realizó con el programa NONA 2.0 (Goloboff 1997), por medio de búsquedas heurísticas, aleatorizando las cuencas con 100 réplicas. El árbol de consenso estricto se determinó con el programa Winclada (Nixon 2000). Se consideraron como áreas de endemismo aquellas ramas terminales del cladograma que contaron con al menos dos especies "sinapomórficas".

\section{Análisis de patrones latitudinales de diversidad}

Los patrones latitudinales de diversidad fueron analizados considerando el número de todas las especies de Plecoptera recolectados en cada una de las cuencas estudiadas (Colwell \& Lees 2000).

Para evitar los sesgos temporales y/o espaciales en la estimación de la diversidad de especies (riqueza específica) de Plecoptera en las áreas estudiadas, así como por diferencias asociadas a la intensidad de los muestreos, dadas las disimilitudes en el número de sitios considerados en cada una de ellas, se utilizó el estimador no paramétrico Chao 2 basado en incidencias (Chao 2005). Con este estimador se determinó la riqueza específica media y su varianza. Para ello se utilizó el programa EstimateS versión 8.0 (Colwell 2005), empleando 1,000 iteraciones con reemplazo de muestras. Para evaluar el efecto del hábitat disponible sobre la diversidad de especies (efecto especies-área, ver Rosenzweig \& Sandlin 1997, Rohde 1999), se analizaron las superficies totales de las cuencas hidrográficas digitalizando cartas a escala 1:250,000 del Instituto Geográfico Militar (Chile). Dada la escala gruesa de estas cartas y del error de medida asociado, los valores de áreas debieran ser considerados como relativos a lo largo de la Patagonia chilena y no como medidas absolutas. Para analizar el efecto de las entradas de energía solar sobre la diversidad de especies (hipótesis especies-energía, ver Currie 1991, Roy et al. 1998, 2000), se determinó 

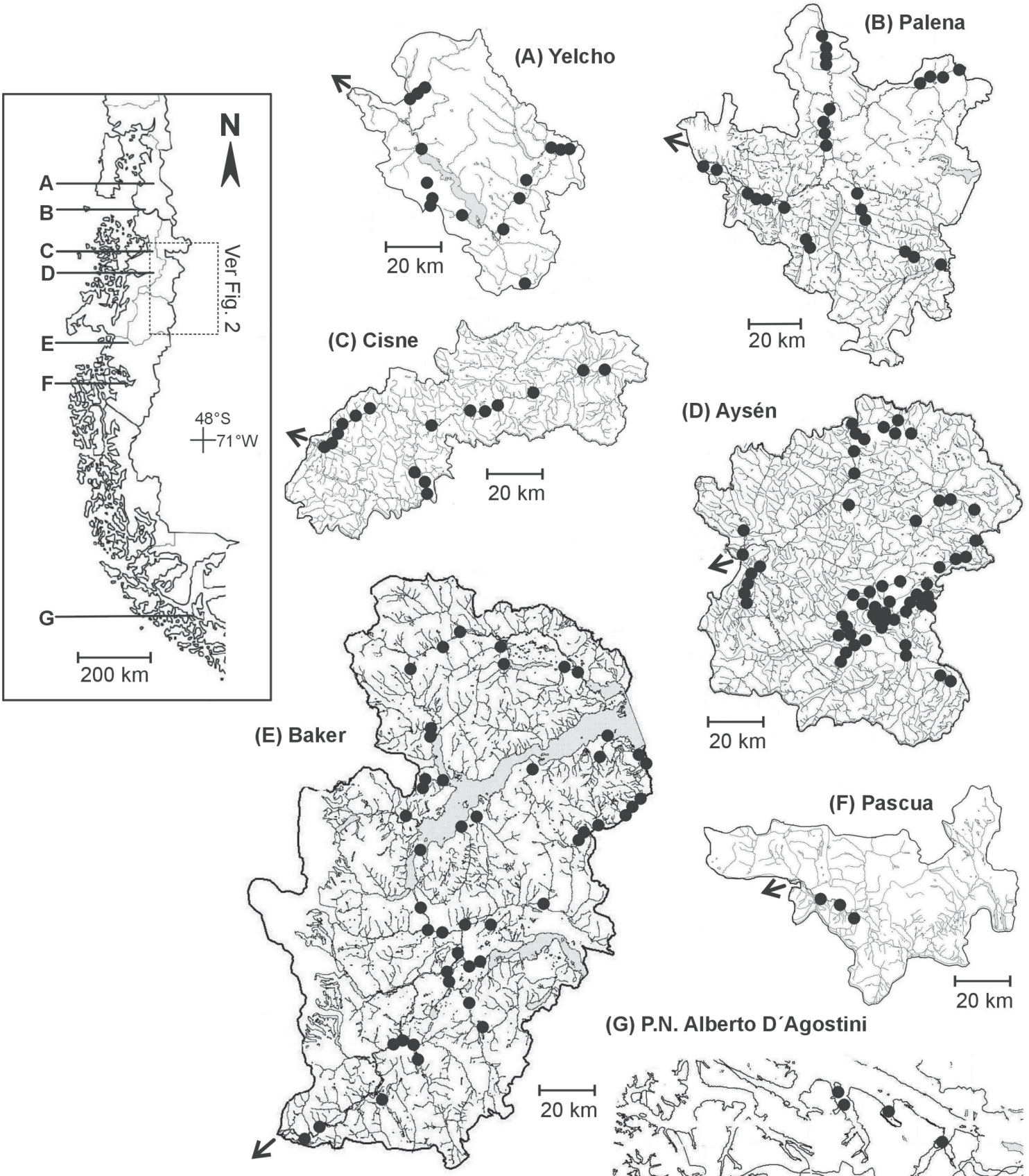

(G) P.N. Alberto D’Agostini

(F) Pascua
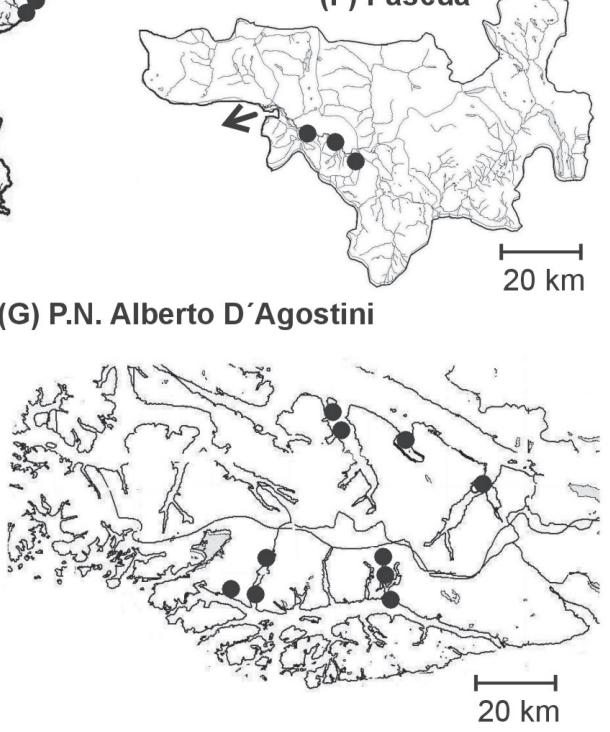

Fig. 1: Vista general del área de estudio en la Patagonia chilena y localización de las siete cuencas estudiadas (A-G). Los círculos negros indican la localización de las 183 estaciones de muestreo. Las flechas señalan la desembocadura de los ríos a la zona costera.

General view of the study area in the Chilean Patagonia, and location of the seven studied river basins (A-G). Black circles indicate the location of the 183 sampling stations. Arrows indicate the discharges from the rivers to the coastal zone. 
la temperatura media anual del aire estimada para el centroide de cada una de las cuencas, sobre la base de las estadísticas de temperatura publicadas por INE (2006).

Para evaluar la regla de Rapoport (Rapoport 1982, Stevens 1989, Roy et al. 1994, Rhode 1999) se empleó la información sobre la distribución geográfica de los Plecoptera generada en el presente estudio, complementada con la existente en la literatura para el PNAA (Moorman et al. 2006), asumiendo un rango de distribución continuo de las especies entre sus límites norte y sur (Colwell \& Lees 2000). Para analizar el posible efecto del tamaño corporal y envergadura alar sobre la distribución de los Plecoptera (ver Malmqvist 2000), se consideró información morfométrica de los estados adultos publicados en la literatura científica, complementada con medidas directas de ejemplares depositados en MZUC.

\section{Análisis del patrón de anidamiento}

Para determinar si las especies de Plecoptera de las siete áreas estudiadas se organizan como subconjuntos anidados del total de la diversidad del área estudiada, se analizaron los patrones de anidamiento de las especies empleando la métrica $\mathrm{T}^{\circ}$ (temperatura del sistema), generada por el programa Nestcalc (Atmar \& Patterson 1993). Esta métrica determina una medida estándar del grado de desorden que presumiblemente se genera en un proceso de colonización-extinción de especies, a partir de la desviación, respecto de una matriz perfectamente anidada, de una matriz con datos de presencia-ausencia de especies (Atmar \& Patterson 1993). Los valores de $\mathrm{T}^{0}$ se distribuyen entre 0 (anidamiento perfecto) hasta 100 (completamente no anidada). La significancia estadística de la matriz se evaluó contrastando la $\mathrm{T}^{\mathrm{o}}$ medida, con 500 matrices nulas que preservan el tamaño y llenado, pero con distribución al azar de presencias y ausencias.

\section{RESULTADOS}

\section{Composición y diversidad de especies}

En todos los sitios de muestreo y cuencas hidrográficas estudiadas, los macroinvertebrados bentónicos de alta vagilidad (Plecoptera), caracterizados por poseer estados adultos alados, fueron notoriamente más diversos que aquellos de baja vagilidad (Aeglidae), lo cual es esperable considerando lo descrito en la literatura (ver Fig. 3 de Valdovinos 2008). La composición y diversidad de especies expresada como riqueza, fue la siguiente:

Macroinvertebrados de alta vagilidad: en el conjunto de 183 sitios de muestreo se registró un total de 30 taxa de Plecoptera, de las cuales 25 fueron identificadas a nivel de especie. Ellas pertenecieron a 25 géneros incluidas en seis familias (Tabla 1). La familia más diversa fue
Gripopterygidae Enderlein, 1909, con 13 géneros y 16 especies, mientras que las familias Notonemuridae Zwick, 1973, Austroperlidae Tillyard, 1921, Perlidae Latreille, 1802 y Diamphipnoidae Ricker, 1950, presentaron entre dos y cuatro géneros y entre dos y cinco especies. Dentro de la familia Eustheniidae Tillyard, 1921, solo se registró la especie Neuroperla schedingi (Navás, 1929). La riqueza específica promedio y su varianza de especies de Plecoptera por cuenca hidrográfica, determinado mediante el estimador Chao2, fue la siguiente: río Yelcho $26.3 \pm 1.8(\mathrm{~N}=18)$, río Palena $33.1 \pm 1.8(\mathrm{~N}=$ $29)$, río Cisnes $20.1 \pm 1.5(\mathrm{~N}=22)$, río Aysén $24.6 \pm 1.2(\mathrm{~N}=50)$, río Baker $22.5 \pm 0.8(\mathrm{~N}=$ $52)$, río Pascua $8.5 \pm 4.0(\mathrm{~N}=3)$, ríos PNAA 6.8 $\pm 2.0(\mathrm{~N}=9)$.

Macroinvertebrados de baja vagilidad: se registraron dos especies de crustáceos aéglidos: a) Aegla alacalufi Jara \& López, 1981, en las cuencas hidrográficas de los ríos Yelcho, Palena y Aysén, y b) Aegla neuquensis Schmitt, 1942, exclusivamente en un sector localizado de la parte alta del río Aysén (Tabla 1). A. alacalufi presentó una amplia distribución dentro del área de estudio, pero restringiéndose siempre en la porción inferior de las cuencas, en la proximidad de la zona de archipiélagos. Por el contrario, A. nequensis se registró solo en la cuenca del río Aysén, específicamente dentro de la subcuenca del río Simpson $\left(45.14^{\circ} \mathrm{S} ; 71.67^{\circ} \mathrm{W}\right)$, en las cercanías de los lagos Castor, Pollux, y en las proximidades de Balmaceda (Fig. 2). A pesar del elevado esfuerzo de muestreo realizado en las cuencas vecinas localizadas al norte y sur de la cuenca del río Aysén, esta especie no fue registrada. No se obtuvieron aéglidos en las cuencas hidrográficas de los ríos Cisne, Baker, Pascua y en los ríos del Parque Nacional Alberto D'Agostini.

\section{Análisis de parsimonia de endemismos}

El análisis permitió identificar un área de endemismo en la cuenca del río Aysén, en la cual se localizan las poblaciones de $A$. neuquensis, la que es muy afín con la cuenca del río Palena, pero no con la del río Cisnes ubicada geográficamente entre estas dos cuencas. Se obtuvieron 100 cladogramas igualmente parsimoniosos de 66 pasos, 


\section{TABLA 1}

Listado de especies recolectadas, clasificadas según la capacidad de dispersión de los estados adultos. Para cada taxon se indica las cuencas hidrográficas en las cuales fueron registradas. Las cuencas son las siguientes (ordenadas de norte a sur) $: \mathrm{Yel}=$ río Yelcho, $\mathrm{Pal}=$ río Palena, $\mathrm{Cis}=$ río Cisnes, Ays = río Aysén, Bak = río Baker, Pas = río Pascua, PNAA = ríos del Parque Nacional Alberto D’Agostini.

List of species collected, classified according to the ability of dispersion of adult stages. For each taxon it is shown the hydrographic river basins in which they were recorded. The river basins are the following (North to South): Yel = Yelcho river, $\mathrm{Pal}=\mathrm{Palena}$ river, $\mathrm{Cis}=$ Cisnes river, $\mathrm{Ays}=$ Aysén river, $\mathrm{Bak}=\mathrm{Baker}$ river, $\mathrm{Pas}=\mathrm{Pascua}$ river, $\mathrm{PNAA}=$ rivers of Parque Nacional Alberto D’Agostini.

\begin{tabular}{llll}
\hline Vagilidad & Familia & Especie o género & Cuencas hidrográficas \\
\hline
\end{tabular}

Baja Crustacea / Malacostraca

Aeglidae Dana, 1852

Perlidae Latreille, 1802

Eustheniidae Tillyard, 1921

Diamphipnoidae Ricker, 1950

Austroperlidae Tillyard, 1921

Gripopterygidae Enderlein, 1909

Insecta / Plecoptera

Notonemouridae Ricker, 1950
Aegla alacalufi Jara \& López, 1981

Aegla neuquensis Schmitt, 1942

Austronemoura chilena Aubert, 1960 Neofulla sp.

Neonemura barrosi Navás,1919

Neonemura sp.

Udamocercia sp.

Kempnyella genualis Illies, 1964

Inconeuria porteri Navás, 1920

Pictetoperla gayi (Pictet, 1841)

Neuroperla schedingi (Navás, 1929)

Diamphipnoa helgae Illies, 1960

DIamphipnopsis samali Illies, 1960

Klapopteryx armillata Navás, 1928

Klapopteryx kuscheli Illies, 1960

Penturoperla barbata Illies, 1959

Antarctoperla altera Zwick, 1973

Antarctoperla michaelseni (Klapelek, 1904)

Antarctoperla sp.

Araucanioperla sp.

Aubertoperla illiesi (Fröehlich, 1960)

Ceratoperla fazi Navás, 1934

Ceratoperla schwabei Illies, 1963

Chilenoperla brundini Illies, 1963

Limnoperla jaffueli (Navás, 1928)

Notoperla archiplatae (Illies, 1958)

Notoperla fuegiana (Enderlein, 1905)

Notoperlopsis femina (Illies, 1963)

Pelurgoperla personata Illies, 1963

Potamoperla myrmidon (Mabille, 1891)

Rhithroperla rossi (Fröehlich, 1960)

Senzilloides panguipulli (Navás, 1928)
Yel, Pal, Ays

Ays

Ays

Yel, Pal, Cis, Ays, Bak

Yel, Pal, Ays, Bak

Pal, Ays

Pal, Bak

Yel, Pal, Cis, Ays, Bak,Pas, PNAA

$$
\begin{aligned}
& \text { Pal, Ays } \\
& \text { Yel, Pal }
\end{aligned}
$$

Yel, Pal, Cis, Ays

Yel, Pal, Cis, Ays

Yel, Pal, Cis, Ays

Yel, Pal, Cis, Ays, Bak

Pal, Bak

Yel, Pal, Cis, Ays, Bak, Pas

Yel, Pal, Cis, Ays, Bak

Pal

Yel, Pal, Cis, Ays, Bak, PNAA

Bak

Ays

Yel, Pal, Cis, Ays, Bak, Pas Ays

Cis, Ays

Yel, Pal, Ays

Yel, Pal, Cis, Ays, Bak, PNAA

Yel, Pal, Ays, Bak, Pas

Yel, Pal, Cis, Ays, Bak, PNAA

Pal, Cis, Ays, Bak

Yel, Pal, Cis, Ays

Yel, Pal, Cis, Ays

Yel, Pal, Cis, Ays, Bak, PNAA

Yel, Pal, Cis, Ays, Bak, Pas 


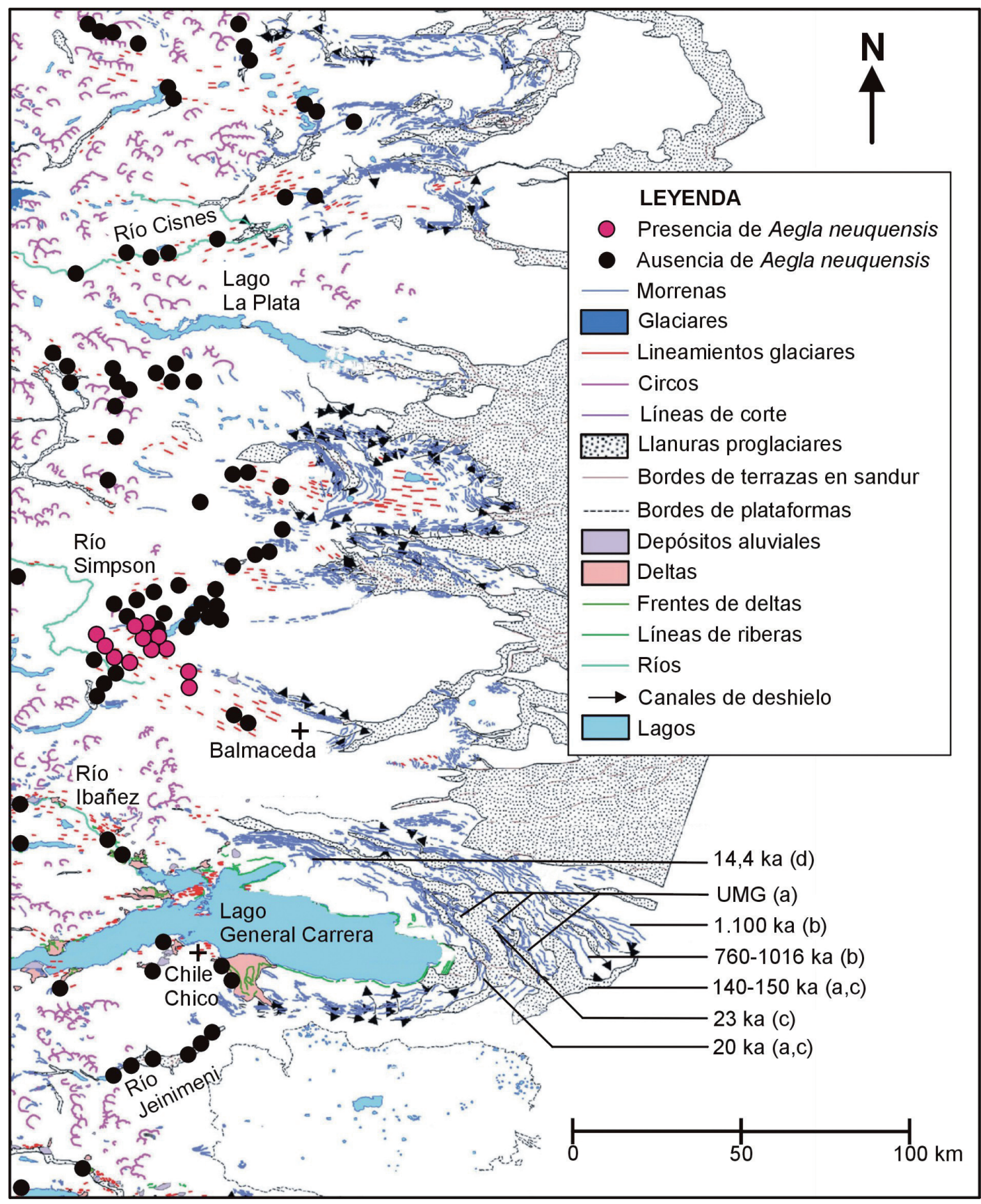

Fig. 2: Distribución espacial del decápodo Aegla neuquensis Schmitt, 1942, en la subcuenca del río Simpson, dentro de la cuenca hidrográfica del río Aysén (círculos rojos). De manera referencial se indican los registros de ausencias de esta especie (círculos negros), en el curso medio-superior de las cuencas comprendidas entre los ríos Palena y Pascua, a lo largo de ca. $340 \mathrm{~km}$ de territorio. Los sitios de muestreo han sido graficados sobre la carta de geomorfología glacial modificada de Glasser et al. (2008). Se adjuntan las fechas de expansión de hielo en el lago General Carrera (lago Buenos Aires) propuestas por: (a) Douglass et al. (2006), (b) Singer et al. (2004) y (c) Kaplan et al. (2005). UMG = Último Máximo Glaciar.

Spatial distribution of the decapod Aegla neuquensis Schmitt, 1942 in the Simpson river sub-basin, within the Aysén river basin (red circles). As a referential aid, the records of species' absences are indicated (black circles), in the upper-half course of the river basins between Palena and Pascua rivers, throughout ca. $340 \mathrm{~km}$ of territory. The sampling sites have been plotted on the glacial geomorphology of the area modified from Glasser et al. (2008). Also shown are the dates of ice expansion in the Lake General Carrera (Buenos Aires Lake) proposed by: (a) Douglass et al. (2006), (b) Singer et al (2004) and (c) Kaplan et al. (2005). UMG = Last Maximum Glacier. 


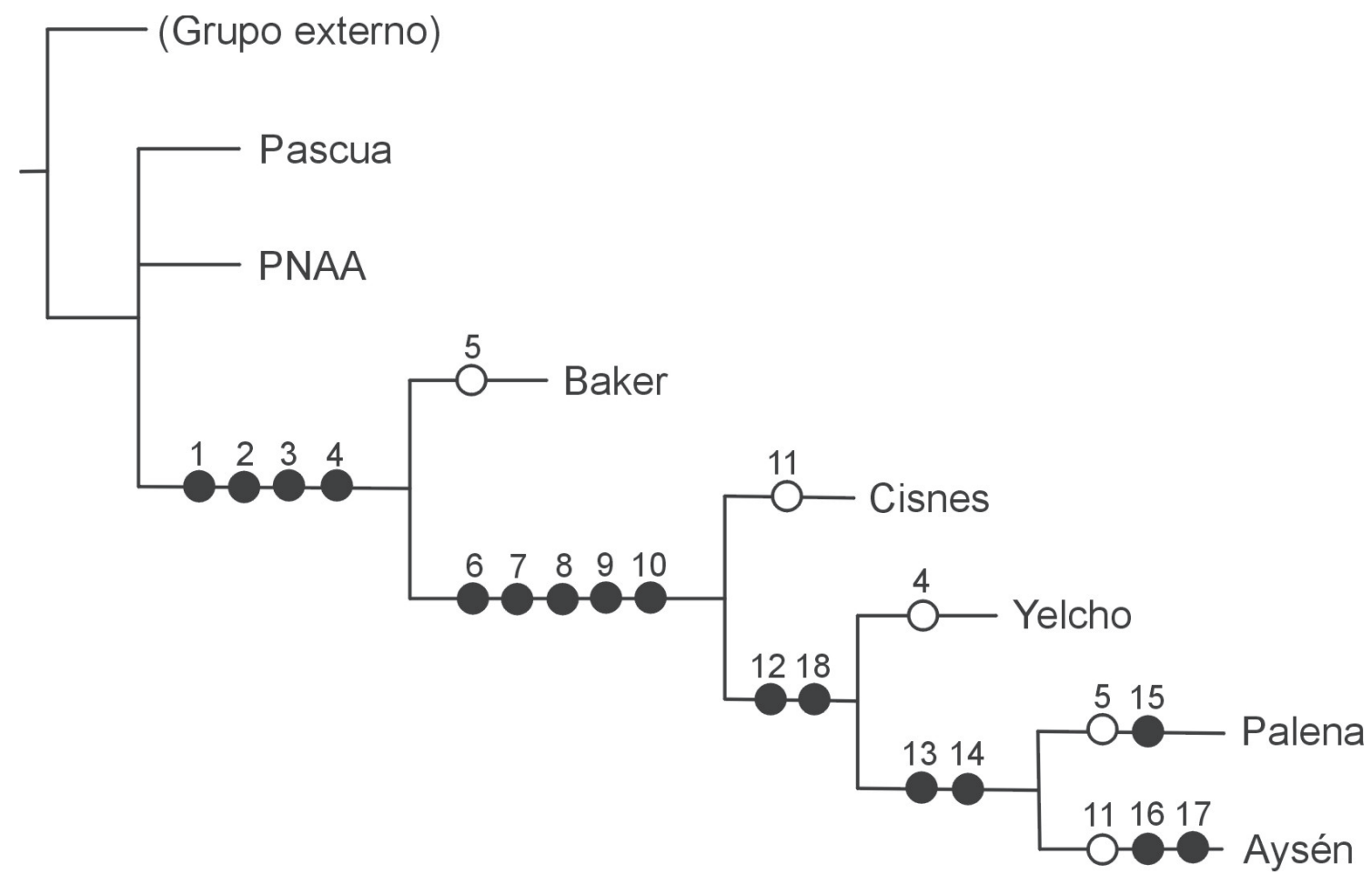

Fig. 3: Árbol de consenso estricto y área de endemismo (cuenca hidrográfica del río Aysén), para estados ninfales de Plecoptera (números 1-16, adultos voladores) y crustáceos Aeglidae (número 17-18, adultos no voladores) en ríos de la Patagonia chilena. $1=$ Austronemoura chilena, $2=$ Diamphipnopsis samali, $3=$ Penturoperla barbata, $4=$ Notoperlopsis femina, $5=$ Klapopteryx armillata, $6=$ Pictoperla gayi, $7=$ Neuroperla schedingi, 8 = Diamphipnoa helgae, $9=$ Pelurgoperla personata, $10=$ Potamoperla myrmidon, $11=$ Ceratoperla schwabei, 12 = Chilenoperla brundini, 13 = Neonemura barrosi, $14=$ Kempnyella genualis, $15=$ Antarctoperla altera, $16=$ Ceratoperla fazi, $17=$ Aegla neuquensis, $18=$ Aegla alacalufi .

Tree of strict consensus and area of endemism (Aysén basin river), for Plecoptera ninphs (numbers 1-16, flying adults) and Aeglidae crustaceans (nonflying, number 17-18, adults) in rivers of the Chilean Patagonia. $1=$ Austronemoura chilena, $2=$ Diamphipnopsis samali, $3=$ Penturoperla barbata, $4=$ Notoperlopsis femina, $5=$ Klapopteryx armillata, $6=$ Pictoperla gayi, $7=$ Neuroperla schedingi, 8 = Diamphipnoa helgae, $9=$ Pelurgoperla personata, $10=$ Potamoperla myrmidon, $11=$ Ceratoperla schwabei, 12 = Chilenoperla brundini, $13=$ Neonemura barrosi, $14=$ Kempnyella genualis, $15=$ Antarctoperla altera, $16=$ Ceratoperla fazi, 17 = Aegla neuquensis, 18 = Aegla alacalufi .

resumidos en un árbol de consenso estricto, a partir del cual se pudo determinar un área de endemismo definido por la presencia de dos sinapomorfías en la cuenca hidrográfica del río Aysén y sustentada por la presencia del plecóptero Ceratoperla fazi Navás, 1934, y del cangrejo A. neuquensis Schmitt, 1942 (Fig. 3). La primera especie restringida a dos sitios de la parte baja de la cuenca, en ríos próximos al lago Riesco con densos bosques lluviosos, y la segunda registrada en 12 estaciones de la parte alta de la subcuenca del río Simpson, en una zona de bosque achaparrado de Lenga (Nothofagus pumilio Poeppig et Endlicher) y pampa patagónica (Fig. 2). En el cladograma se observa que algunas especies están presentes en áreas únicas, como ocurre con Antarctoperla altera Zwick, 1973 en la cuenca del río Palena. Además, se determinó a las especies Pictoperla gayi (Pictet, 1841), Neuroperla schedingi (Navás, 1929), Diamphipnoa helgae Illies, 1960, Pelurgoperla personata Illies, 1963 y Potamoperla myrmidon (Mabille, 1891), como las especies sinapomórficas del mayor número de las cuencas hidrográficas estudiadas (Yelcho, Palena, Cisnes y Aysén), quedando fuera las cuencas de los ríos Baker y Pascua, y los ríos del Parque Nacional Alberto D’Agostini. Las especies Kempnyella genualis Illies, 1964, y Neonemoura barrosi Navás, 1919, fueron sinapomórficas en las cuencas de los ríos 
Palena y Aysén, pero no en las restantes, incluyendo a la cuenca del río Cisnes localizada geográficamente inmediatamente entre ellas. Las especies Aegla alacalufi y Chilenoperla brundini Illies, 1963, forman parte del conjunto Yelcho - Palena - Aysén, pero estando ausentes en la cuenca del río Cisnes.

\section{Análisis de patrones latitudinales de diversidad}

Dado el escaso número de las especies de baja vagilidad que fueron registrados en el área de estudio $(\mathrm{N}=2)$, el análisis de los patrones latitudinales de diversidad se focalizó en las de alta vagilidad.

Un marcado cambio latitudinal en la diversidad de especies de Plecoptera fue evidente a lo largo de ca. $1,400 \mathrm{~km}$ de la Patagonia chilena, entre Chaitén $\left(42^{\circ} 50^{\prime} \mathrm{S}\right)$ y el Parque Nacional Alberto D'Agostini (PNAA, $54^{\circ} 43^{\prime} \mathrm{S}$ ) (Fig. $4 \mathrm{~A}, \mathrm{R}^{2}=0.72, \mathrm{P}<0.05$ ). Este cambio fue particularmente evidente en el caso de la familia Gripopterygidae, el grupo más diverso de Plecoptera dentro del área de estudio. El patrón latitudinal de diversidad estaría explicando en parte los resultados del Análisis de Parsimonia de Endemismos, en el cual predominan numéricamente las especies móviles. En la Fig. 3 se observa que las cuencas situadas más al norte (Yelcho, Palena, Cisnes y Aysén) tienden a diferenciarse de las localizadas hacia la región austral (Baker, Pascua y PNAA), en relación con cambios en la riqueza de especies de Plecoptera.

Para evaluar el efecto de la superficie de las cuencas estudiadas sobre la diversidad de Plecoptera, se examinó la relación entre riqueza de especies y el área de ellas. Esta regresión no fue estadísticamente significativa (Fig. $4 \mathrm{~B}, \mathrm{R}^{2}=0.24, \mathrm{P}=0.12$ ). Al remover el efecto potencial de la latitud, ello no mejoró la regresión ya que no se observó una relación significativa entre el área de las cuencas y los residuos de la regresión del número de especies versus latitud. A diferencia del área, la temperatura media del aire explicó una porción significativa de la varianza $(67 \%)$ de la diversidad de especies de Plecoptera de la Patagonia chilena. La regresión entre riqueza de especies y la temperatura media anual del aire fue estadísticamente significativa (Fig. 4C, $\left.\mathrm{R}^{2}=0.67, \mathrm{P}<0.05\right)$, lo que sugiere que la hipótesis especies-energía asociada al efecto de las entradas de energía solar puede tener un efecto relevante sobre la diversidad de especies de Plecoptera. El coeficiente de regresión aumentó notablemente al evaluar la relación entre la densidad de taxa existente en cada una de las cuencas (Chao2/área de la cuenca) y la temperatura media anual del aire (Fig. $\left.4 \mathrm{D}, \mathrm{R}^{2}=0.90, \mathrm{P}<0.05\right)$, indicando que sí existiría un cierto efecto de área sobre el patrón latitudinal de Plecoptera, aunque notablemente inferior al de la temperatura. Por otra parte, al estudiar la relación existente entre densidad de taxa y latitud, se constató que este comportamiento no es monotónico, presentando una tendencia lineal entre las cuencas del río Yelcho y Pascua (Fig. 4E, $\mathrm{R}^{2}=$ $0.90, \mathrm{P}<0.05)$, para luego presentar un fuerte quiebre hacia el sur en dirección a Tierra del Fuego (PNAA). Este punto de quiebre es coincidente con un cambio del régimen térmico observado en el área de los campos de hielo Norte y Sur, a la latitud de las cuencas de los ríos Baker y Pascua (Fig. $4 \mathrm{~F}, \mathrm{R}^{2}=0.95, \mathrm{P}<$ 0.05).

Para evaluar la regla de Rapoport, se examinó la relación entre la latitud media del rango latitudinal de todos los taxa identificados hasta nivel de especie y el rango latitudinal expresado en kilómetros. Esta regresión fue estadísticamente significativa (Fig. $5 \mathrm{~A}, \mathrm{R}^{2}=$ $0.64, \mathrm{P}<0.05)$, mostrando un claro incremento de los rangos latitudinales de las especies, a medida que ellas se localizan más al sur de la Patagonia, a pesar la marcada disminución de la riqueza de especies en esta misma dirección. Ello permite pensar que las especies con rangos medios de distribución localizados más al sur, tendrían mayores rangos de tolerancia de temperatura que aquellas especies con rangos medios localizados más al norte, que es el área con una mayor riqueza de especies de Plecoptera. Las especies con mayor rango latitudinal de distribución fueron Potamoperla myrmidon (Mabille, 1891) y Limnoperla jaffueli (Navás, 1928), mientras que las con un menor rango fueron Neuroperla schedingi (Navás, 1929) y Klapopteryx armillata (Navás, 1928). No se observó asociación significativa entre el tamaño corporal (Fig. 5B) y la longitud alar (Fig. 5C) de las especies de Plecoptera con su rango latitudinal. Tampoco entre la proporción entre la envergadura alar y el tamaño corporal (E:T), y la latitud media de 
(A)

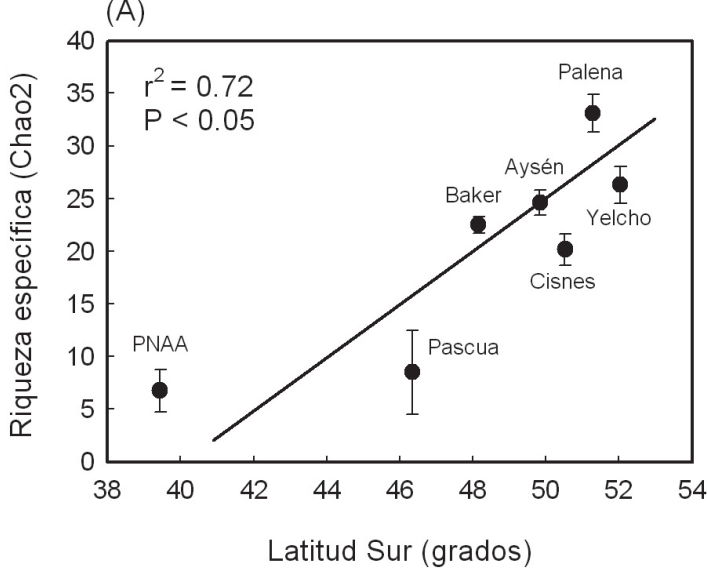

(C)

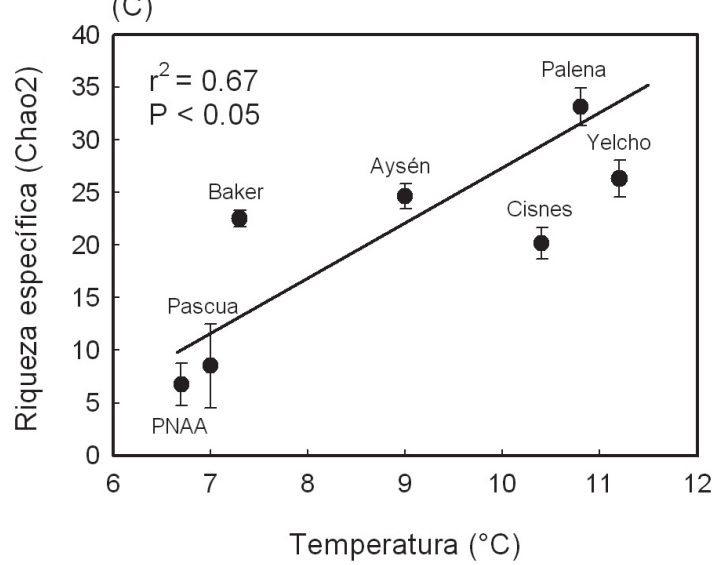

(E)

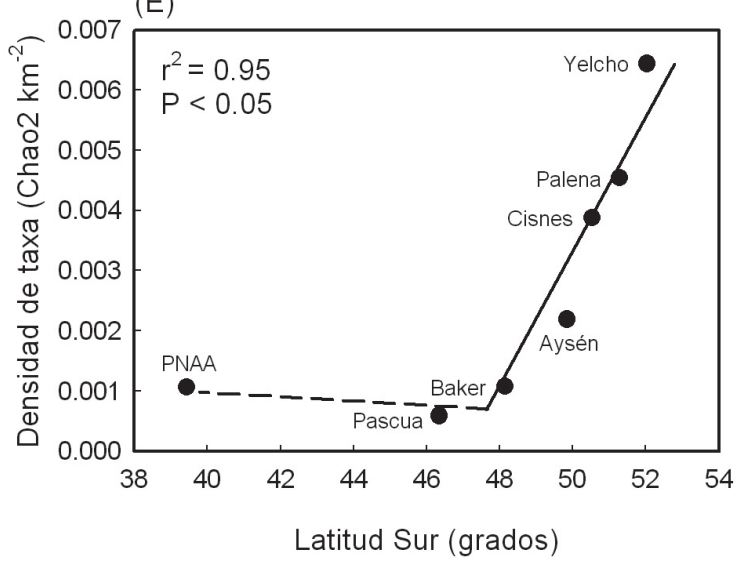

(B)

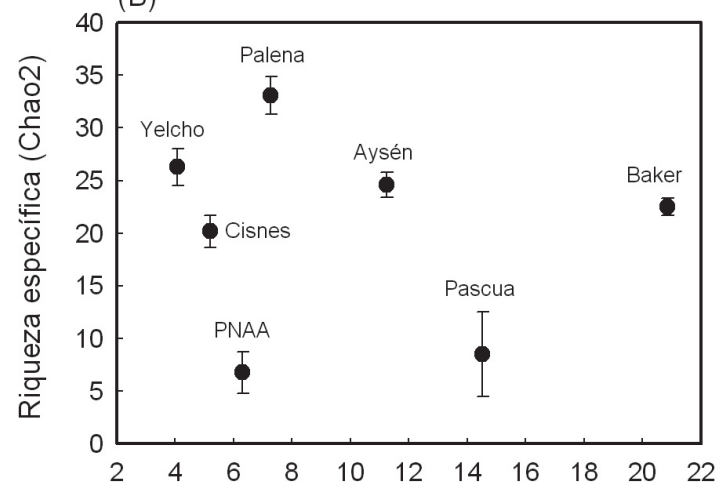

Área de la cuenca $\left(\mathrm{km}^{2} \times 1,000\right)$

(D)

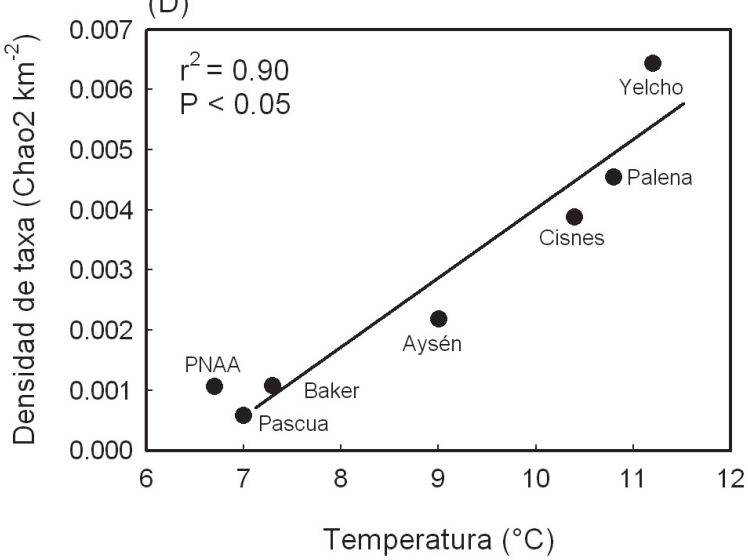

$(\mathrm{F})$

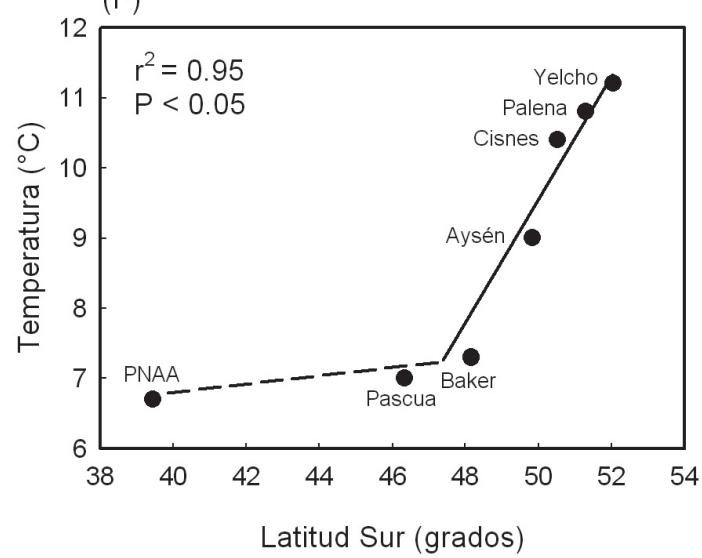

Fig. 4: Diagramas de dispersión entre variables biológicas (riqueza específica y densidad de taxa de Plecoptera) y ambientales (temperatura media del aire, área de la cuenca y latitud), de las siete cuencas hidrográficas estudiadas en la Patagonia chilena. (A) Riqueza específica (S) v/s latitud (L), (B) S v/s área de la cuenca, (C) $\mathrm{S} v / \mathrm{s}$ temperatura media del aire (T), (D) densidad de taxa (D) v/s T, (E) D v/s L, (F) T v/s L. Para la riqueza específica se muestra la media y varianza de los estimadores de Chao2.

Scatter diagrams among biological (species richness and density of taxa of Plecoptera) and environmental variables (average air temperature, area of the river basin, and latitude), of the seven studied hydrographic river basins in the Chilean Patagonia. (A) Species richness (S) v/s latitude (L), (B) S v/s basin area, (C) S v/s mean air temperature (T), (D) density of taxa (D) v/s T, (E) D v/s L, (F) T v/s L. For species richness, the average and variance of the Chao2 estimators are indicated. 
(A)

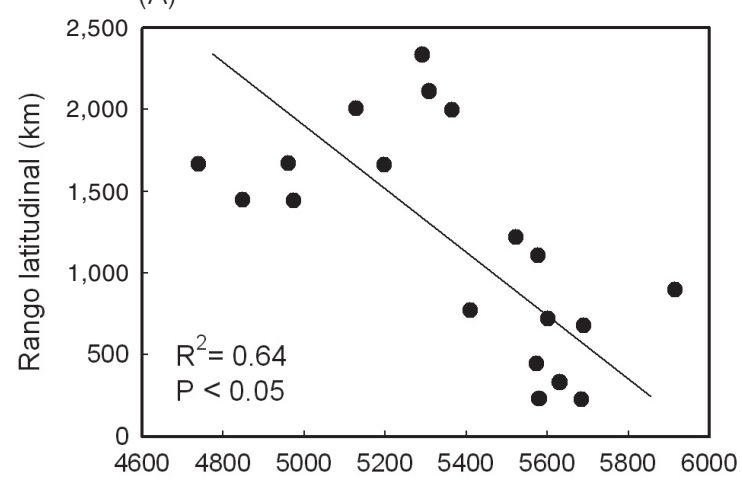

(C)

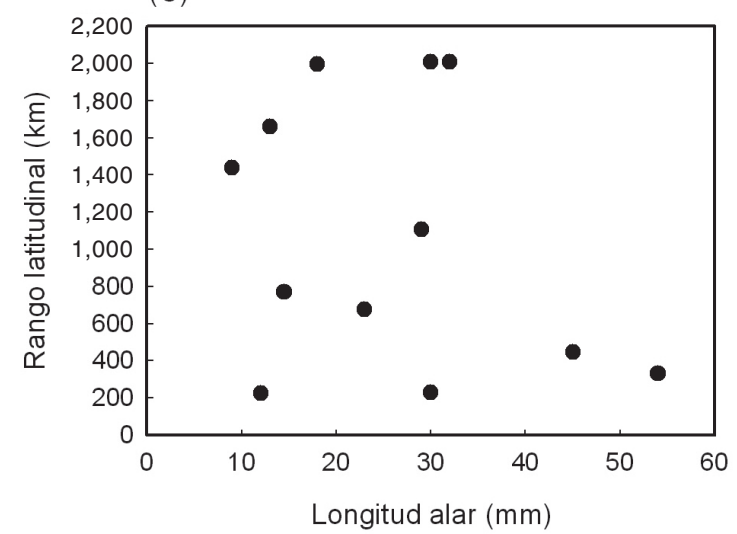

(E)

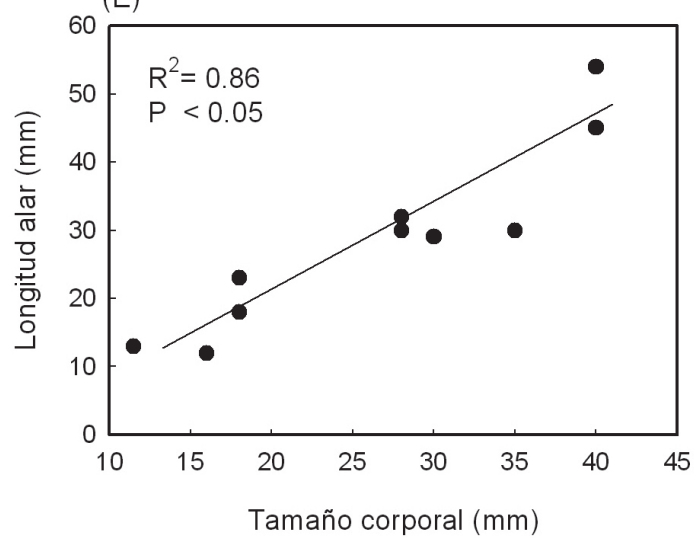

(B)

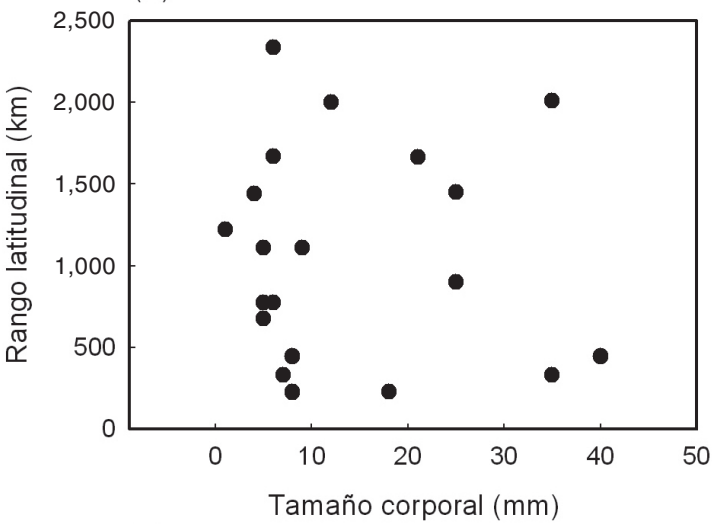

(D)

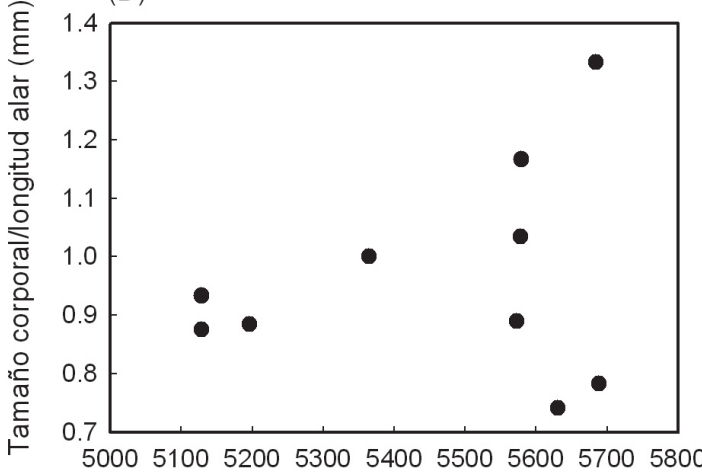

$(\mathrm{F})$

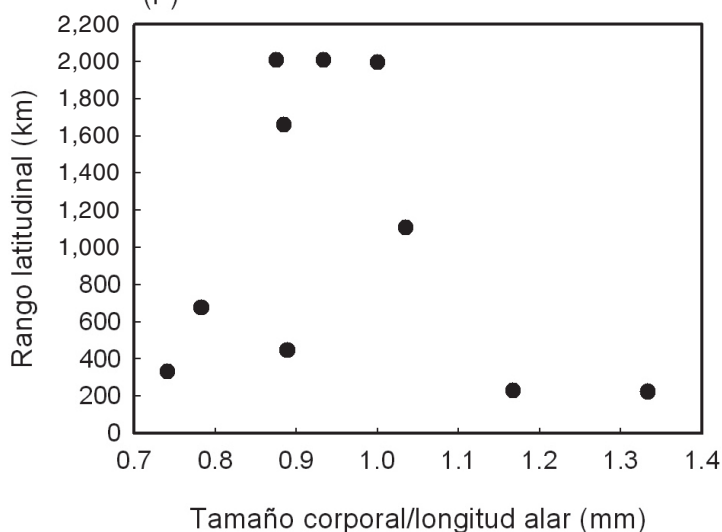

Fig. 5: Diagramas de dispersión entre variables biológicas de los Plecoptera presentes en las siete cuencas estudiadas de la Patagonia chilena (rango latitudinal, latitud media, tamaño corporal de adultos, longitud alar y proporción tamaño corporal/longitud alar), que fueron identificados hasta nivel de especie y que cuentan con información morfométrica de los estados adultos. (A) Rango latitudinal (RL) v/s latitud media (LM), (B) RL v/s tamaño corporal (TC), (C) RL v/s longitud alar (LA), (D) TC/LA v/s LM, (E) LA v/s TC, (F) RL v/s TC/LA.

Scatter diagrams among biological variables of Plecoptera from the seven studied river basins of the Chilean Patagonia (latitudinal range, mean latitude, adult body size, wing size, and body size/wing size ratio), that were identified to the species level, and for which there are available morphometric information of the adults. (A) Latitudinal range (RL) v/s mean latitude (LM), (B) RL v/s body size (TC), (C) RL v/s wing length (LA), (D) TC/LA v/s LM, (E) LA v/s TC, (F) RL v/s TC/LA. 
los taxa (Fig. 5D), lo cual es de esperar considerando la clara relación linear existente entre envergadura y el tamaño corporal de los adultos (Fig. 5E, $\mathrm{R}^{2}=0.86, \mathrm{P}<0.05$ ). La proporción E:T tampoco mostró una asociación estadísticamente significativa con el rango latitudinal de las especies (Fig. 5F).

\section{Patrón de anidamiento de Plecoptera}

Las especies de Plecoptera de las siete áreas estudiadas presentaron un patrón de anidamiento altamente significativo $(\mathrm{P}<0.01)$, implicando una distribución no azarosa en subconjuntos de especies. La matriz resultante tuvo un grado de relleno de un $57.1 \%$ y presentó una temperatura de $13.4^{\circ}$ que fue $36.2^{\circ}$ más baja que la esperada por azar $\left(\mathrm{T}^{\circ}=\right.$ $\left.49.6^{\circ} \pm 7.1 ; \mathrm{N}=500\right)$. En la matriz anidada de Fig. 6 se observa una clara distribución nortesur de las cuencas estudiadas (de Palena a PNAA). Esta sucesión de cuencas es la misma mostrada en la Fig. 4A que relaciona riqueza específica con latitud, explicada principalmente por cambios en la temperatura del aire (Fig. 4B).

\section{DISCUSIÓN}

Los patrones de distribución geográfica determinados para los taxa de Plecoptera y Aeglidae estudiados forman parte de cuatro tipos de regiones "oceánicas” (i.e., influencia mediterránea, temperada-fría, transandina, subantártica), más la "continental andina", sensu Di Castri (1968). Estas regiones representan zonas ecológicas y geomorfológicas particulares, definidas por formaciones vegetacionales asociadas a determinadas condiciones latitudinales y altitudinales. La marcada variabilidad latitudinal en los regímenes térmicos que existen en el sur de Chile, sería uno de los factores que determinarían la composición y riqueza de especies de Plecoptera en cuencas hidrográficas especificas. Sin embargo, esta situación no sería válida para el caso de $A$. nequensis que se encuentra restringida a la parte alta de la cuenca hidrográfica del río Aysén y que correspondería a una población relictual probablemente derivada de Aegla abtao riolimayana Schmitt, 1942, distribuida en los ríos de la estepa argentina localizados hacia el este de la cuenca del río Aysén. Hasta el Último Máximo Glacial (UMG), A. abtao riolimayana se habría distribuido en la cuenca del río Mayo que drena hacia el Atlántico. Sin embargo, debido a la deglaciación de la zona superior de esta cuenca, habría sido “capturada" por la cuenca del río Aysén cambiando su drenaje hacia el Pacífico, quedando estas poblaciones aisladas geográficamente de las restantes que hoy existen en la Patagonia argentina, originando a A. neuquensis (Mardones et al., datos no publicados).

En el cladograma obtenido se observan las divergencias distribucionales de las especies estudiadas. El patrón de áreas anidadas (Fig. 3) permitió determinar un área de endemismo, en la cuenca hidrográfica del río Aysén, por la presencia del cangrejo $A$. neuquensis y del Plecoptera Ceratoperla fazi. Esta última especie estaba previamente descrita solo para territorios chilenos y argentinos localizados entre $\operatorname{los} 38^{\circ}-44^{\circ} \mathrm{S}$ (Illies 1963, Vera \& Camousseight 2006). El área de Aysén es muy afín con la cuenca del río Palena, pero no con la del río Cisnes localizada entre estas dos. Los resultados del cladograma serían explicados por diversos factores, que habrían afectado diferencialmente los taxa de alta y baja vagilidad. En el caso de los primeros, los acontecimientos históricos parecieran tener menos influencia que las exigencias ecológicas actuales, así como ocurre en muchos insectos con una fase de dispersión aérea (Vinson \& Hawkins 2003). Sin embargo, en el caso de los de baja vagilidad, los fenómenos históricos tendrían mayor relevancia. Al respecto, los patrones de distribución espacial actuales de los taxa estudiados y la presencia del área de endemismo podrían ser explicados fundamentalmente por dos vías:

Macroinvertebrados de baja vagilidad: dada la ausencia de formas de dispersión aladas, los crustáceos Aeglidae presentan proporcionalmente una escasa capacidad de dispersión en relación a los Plecoptera. Otro factor limitante a la dispersión de estos organismos está asociado a su aparentemente escasa capacidad de osmorregulación, lo que les impide dispersarse entre cuencas a través de las aguas marinas costeras. En el hecho, no hemos observado Aeglidae en aguas con 
salinidades superiores a 2 UPS, a diferencia del crustáceo Hemigrapsus crenulatus (MilneEdwards, 1837), que está ampliamente distribuido en los fiordos patagónicos (Valdovinos 2004). Dados estos dos factores, la actual distribución de estos organismos estaría relacionada fundamentalmente por la mantención de su distribución ancestral en áreas no glaciadas en el Plesitoceno, que actuaron como áreas refugiales para estos organismos. Probablemente, a partir de estas áreas ellos hayan incrementado su área de distribución dentro de las cuencas, ocupando hábitats que cumplan con sus requerimientos ecológicos pero limitados por su escasa vagilidad. En la actualidad la especie $A$. alacalufi se distribuye ampliamente en Chile desde Chiloé hasta la isla Madre de Dios (Jara et al. 2006), y en este estudio se registró específicamente en la parte baja de las cuencas de los ríos Yelcho, Palena y Aysén. La distribución de esta especie sugeriría que estas zonas no estuvieron glaciadas en el Pleistoceno o que fueron recolonizadas desde determinadas áreas refugiales, a través de un gran "corredor" dulceacuícola que se habría formado durante el rápido deshielo de los glaciales, considerando que hay claras evidencias de un marcado descenso en la salinidad de la zona de los fiordos (ver De PolHolz et al. 2006). La historia de A. neuquensis habría sido distinta. En la actualidad esta especie solamente se encuentra distribuida en la parte alta de la cuenca del río Aysén (Fig. 2). Antecedentes sobre la geomorfología glacial del área desarrollados por Mardones et al. (datos no publicados) en el marco de este estudio, indican que esta disyunción estaría asociada a un cambio de orientación de flujo de la red hídrica (captura hidrológica), ocurrida en la parte alta de la cuenca del río Aysén derivada del retroceso glaciar, que habría dejado aislada a una población que originalmente formaba parte del río Mayo de la estepa argentina. Hay evidencias geomorfológicas que indican que parte de esta zona que actualmente corresponde a la cuenca del río Aysén (i.e., lago Thompson) no estuvo cubierta por hielo en el Último Máximo Glacial (UMG). En la Fig. 2 se muestra la

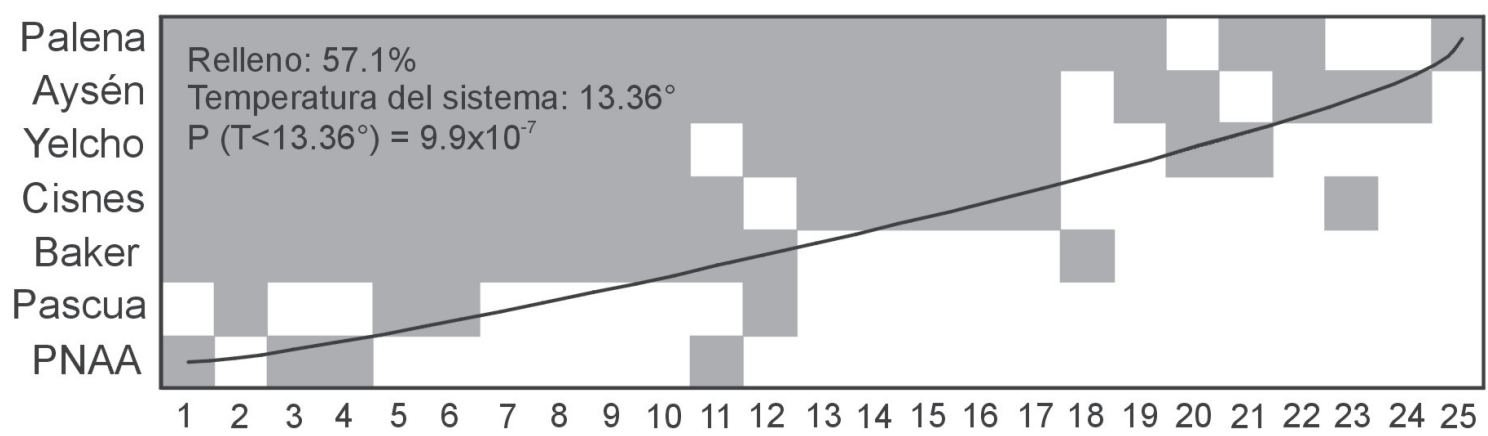

Fig. 6: Matriz anidada de especies de Plecoptera de las siete cuencas estudiadas de la Patagonia chilena. Celdas grises y blancas corresponden a presencias y ausencias, respectivamente. La línea sólida corresponde a la isolínea de perfecto anidamiento. Numeración de las especies: 1 Rhithroperla rossi, 2 Aubertoperla illiesi, 3 Antarctoperla michaelseni, 4 Limnnoperla jaffueli, 5 Klapopteryx kuscheli, 6 Senzilloides panguipullii, 7 Penturoperla barbata, 8 Austronemoura chilena, 9 Notoperla fungiana, 10 Diamphipnopsis samali, 11 Notoperlopsis femina, 12 Notoperla archiplatae, 13 Diamphipnoa helgae, 14 Pictoperla gayi, 15 Pelurgoperla personata, 16 Neuroperla schedingi, 17 Potamoperla myrmidon, 18 Klapopteryx armillata, 19 Kempnyella genualis, 20 Chilenoperla brundini, 21 Inconeuria porteri, 22 Neonemura barrosi, 23 Ceratoperla schwabei, 24 Ceratoperla fazi, 25 Antarctoperla altera.

Nested matrix of species of Plecoptera, of the seven studied river basins of the Chilean Patagonia. Gray and white cells correspond to presences and absences respectively. Solid line represents the isoline of perfect nestedness. Number of the species: 1 Rhithroperla rossi, 2 Aubertoperla illiesi, 3 Antarctoperla michaelseni, 4 Limnnoperla jaffueli, 5 Klapopteryx kuscheli, 6 Senzilloides panguipullii, 7 Penturoperla barbata, 8 Austronemoura chilena, 9 Notoperla fungiana, 10 Diamphipnopsis samali, 11 Notoperlopsis femina, 12 Notoperla archiplatae, 13 Diamphipnoa helgae, 14 Pictoperla gayi, 15 Pelurgoperla personata, 16 Neuroperla schedingi, 17 Potamoperla myrmidon, 18 Klapopteryx armillata, 19 Kempnyella genualis, 20 Chilenoperla brundini, 21 Inconeuria porteri, 22 Neonemura barrosi, 23 Ceratoperla schwabei, 24 Ceratoperla fazi, 25 Antarctoperla altera. 
geomorfología glacial del área comprendida entre $\operatorname{los} 30^{\circ}$ y $45^{\circ} \mathrm{S}$ estudiada por Glasser et al. (2008), incluyendo los sitios de muestreo en el área destacando aquellos en los cuales se registró $A$. neuquensis. En esta zona, los datos de expansión del hielo registrados en el área del lago General Carrera/lago Buenos Aires, indican un UMG hace 23-16 ka (basados en las morrenas de Fénix; Kaplan et al. 2005, Douglass et al. 2006).

Macroinvertebrados de alta vagilidad: los Plecoptera, que son un grupo de elevada capacidad de dispersión por el hecho de poseer estados adultos alados, habrían colonizado vía aérea las zonas deglaciadas, desde diversas zonas refugiales existentes en el área de los fiordos y en el área norte del área de estudio. En la actualidad, estos organismos se ajustan al gradiente térmico existente en el área, con un claro decaimiento monotónico de la diversidad de especies hacia el sur de la Patagonia chilena, lo cual es consistente con los resultados obtenidos por Palma \& Figueroa (2008). Asociado a esto, los rangos latitudinales de las especies se incrementan hacia el sur, lo que sugiere que las especies más australes son más euritérmicas que las de más al norte. No se registró una asociación significativa entre los patrones de distribución espacial y la longitud alar de los adultos. Este decaimiento en la riqueza específica hacia los polos ha sido reportado en numerosos taxa vegetales y animales, tanto terrestres como marinos (e.g., Fischer 1959, Gaines \& Lubchenco 1982, Willig \& Selcer 1989, Currie 1991, Rex et al. 1993, Roy et al. 1994, Blackburn \& Gaston 1996, Valdovinos et al. 2003, Marquet et al. 2004). En el caso de los insectos dulceacuícolas, esta situación también se ha registrado, aunque la información disponible es más escasa (ver Arthington 1990, Vinson \& Hawkins 1998, Boyero 2002, Palma \& Figueroa 2008). En general se asume que los Plecoptera tienen preferencias por aguas frías, siendo ambientalmente muy exigentes (i.e., Theischinger 1991, Figueroa et al. 2003, Fochetti \& Tierno de Figueroa 2007). Sin embargo, los resultados del presente estudio sugieren que las aguas de baja temperatura tienden a limitar la diversidad de Plecoptera (Fig. 4C), por lo que asociado a los ciclos glaciales cabría esperar retracciones y ampliaciones de los rangos latitudinales $\mathrm{y}$ altitudinales en la distribución de las especies. En la actualidad, en el caso de Plecoptera, las exigencias ecológicas presentes parecieran tener más influencia que los acontecimientos históricos del Plesitoceno, al igual que en muchos otros grupos de insectos acuáticos con fases adultas aladas (Vinson \& Hawkins 2003).

Ha existido un amplio debate sobre los mecanismos ecológicos y evolutivos del origen de los gradientes latitudinales de riqueza de especies (Rohde 1992, Brown \& Lomolino 1998, Gaston 2000, Willig et al. 2003). Algunas de las hipótesis incluyen la disponibilidad de energía y condición térmica, o variables tales como heterogeneidad ambiental, área disponible, diferencias en las tasas de especiación o extinción y la consecuencia de las últimas glaciaciones (Sax 2001). La mayoría de los estudios han establecido una asociación empírica, directa o indirecta, entre la riqueza de especies y gradientes climáticos, especialmente con el potencial de evapotranspiración (Currie \& Paquin 1987), temperatura (Turner et al. 1987) y productividad (Kaspari et al. 2000), siendo los dos primeros factores ambientales fuertes predictores de la diversidad acuática y terrestre (Hawkins et al. 2003, Hillebrand 2004). En el caso de sistemas acuáticos dulceacuícolas, numerosos estudios muestran cómo la temperatura afecta de manera importante la diversidad de invertebrados fluviales (Ward \& Stanford 1982, Allan 1995), variable que se encuentra estrechamente asociada a la latitud (Jacobsen et al. 1997). De esta forma, modificaciones en las variables climáticas pueden dar lugar a cambios en la diversidad y rangos de distribución de las especies a través de la latitud (Crozier 2004, Hampe \& Petit 2005, Morrison et al. 2005).

Las especies de Plecoptera de las siete áreas estudiadas, se encuentran organizadas como subconjuntos significativamente anidados $(\mathrm{P}<0.01)$, implicando una distribución no azarosa en subconjuntos de especies. Al respecto, nuestros resultados sugieren que este anidamiento estaría asociado con los distintos requerimientos ecológicos actuales de las especies, tales como la temperatura, y no a las capacidades de dispersión de los adultos. El anidamiento podría ser explicado por los fuertes gradientes 
climáticos existentes en la Patagonia chilena (Di Castri 1968), junto a procesos históricos de colonización-extinción y a fenómenos estocásticos ocurridos dentro de las cuencas estudiadas. El anidamiento también puede ser el resultado de diferencias en las capacidades de dispersión de las especies. Si ellas provienen de una misma área fuente (e.g., refugio glacial). Los buenos colonizadores alcanzarían grandes áreas, mientras que las especies con una menor capacidad de dispersión permanecerán restringidas, lo cual generará un patrón anidado (Patterson \& Atmar 1986, Lomolino 1996, Hausdorf \& Henning 2003). Esto último no es el caso de lo observado en los Plecoptera de la Patagonia chilena, no encontrándose una asociación significativa entre tamaño corporal y longitud alar de los adultos con la latitud media y rango latitudinal de las especies (Fig. 5).

Dado el escaso número de especies de baja vagilidad que fueron estudiados, no fue posible realizar un análisis de anidados con este tipo de organismos. En el caso de los organismos dulceacuícolas que no tienen la capacidad de volar (e.g. crustáceos, moluscos y planarias), la dispersión pasiva decrece con un incremento del tamaño corporal, esperándose que presenten un patrón anidado asociado a su capacidad de dispersión desde una fuente de origen (Hausdorf \& Henning 2003). En el caso de comunidades de plancton presente en lagos chilenos, los resultados de Ramos-Jiliberto et al. (2009) han mostrado que el fitoplancton y zooplancton total, al igual que las asociaciones de diatomeas, copépodos y cladóceros, están altamente anidados, siendo el área superficial y el grado de aislamiento de los lagos las variables que con mayor probabilidad explican tal anidamiento.

Los resultados del presente estudio, a pesar de haber considerado solo dos grandes grupos taxonómicos (Plecoptera y Aeglidae), corresponden al primer análisis comparativo de la biodiversidad de macroinvertebrados dulceacuícolas de las principales cuencas de la Patagonia chilena. La mayoría de los estudios biogeográficos realizados para insectos de Sudamérica, han estado dirigidos a comparar la similitud biótica entre la región Neotropical y distintas áreas del mundo, basándose en taxa compartidos con los restantes continentes australes (Kuschel 1964, 1969, Morrone et al.
1994). Autores como Peña (1966), Morrone et al. (1997, 2002), Donato et al. (2003), PizarroAraya \& Jerez (2004) han realizado estudios utilizando taxa propios de Sudamérica austral, principalmente Coleoptera terrestres, mientras que Morrone (1996) y Roig-Juñent et al. (2003) realizaron análisis biogeográficos utilizando artrópodos epígeos.

En general, los estudios que han incluido la biota dulceacuícola de la Patagonia chilena son escasos, pudiéndose citar solo los trabajos de Dyer (2000) y Ruzzante et al. (2008), quienes se centraron en el estudio de la diversidad de peces, y de Pérez-Lozada et al. (2002a, 2002b), los cuales estudiaron los crustáceos anomuros del género Aegla y de Oyanedel et al. (2008), quienes estudiaron la biota pelágica de diversas cuencas a lo largo de Chile (fitoplancton, zooplancton y peces). E1 conocimiento actual de áreas de endemismo en Chile se reduce a los aportes de Posadas (1996) para la XII Región, Squeo et al. (1998) y Cavieres et al. (2002) para la II Región, y Cavieres et al. (2001) para la IV Región de Chile, quienes establecen para la vegetación áreas de concentración de especies endémicas. En términos faunísticos, Jerez (2000) y Pizarro-Araya \& Jerez (2004) delimitan áreas de endemismo para la II Región en base a la fauna de Coleópteros terrestres, y Oyanedel et al. (2008), quienes identificaron cuatro áreas de endemicidad para peces, y numerosas otras para Rotifera, Copepoda y Cladocera. El presente estudio representa el primer intento de identificar áreas de endemismo mediante PAE, considerando los macroinvertebrados bentónicos presentes en los ecosistemas dulceacuícolas fluviales chilenos.

Los patrones de distribución espacial de la biota de los ríos presentan una elevada complejidad, debido a que los paisajes fluviales incluyen una gran cantidad de series de hábitats y gradientes ambientales (Ward 1998). Estos tipos de sistemas son altamente dinámicos, manifestando constantemente cambios en los hábitats (Stanford et al. 1996, Ward et al. 2002), lo que los hace sistemas complejos y diversos, muy sensibles a las perturbaciones generadas por el hombre (Bornette et al. 1998, Ward 1998). En los ecosistemas fluviales chilenos, los estados ninfales de Plecoptera y Aeglidos constituyen importantes componentes ecológicos de 
sistemas de aguas frías, y bien oxigenadas. A escala local, factores tales como temperatura, altitud, tipo de sustrato de fondo, velocidad de la corriente, clima y tipo de vegetación ribereña son los principales determinantes de su distribución (Miserendino 2006, Theischinger 1991, Figueroa et al. 2003, Oyanedel et al. 2008). El conocimiento sobre la ecología de los estados ninfales de Plecoptera de Sudamérica austral se ha incrementado notablemente en la última década. Estos se han enfocado principalmente: a) distribución en gradientes altitudinales (Albariño 1997, Miserendino \& Pizzolon 2000), b) fluctuaciones espacio-temporales y su relación con las variables ambientales (Miserendino 2006), c) estudios sobre la dieta de diferentes especies (Díaz-Villanueva \& Albariño 1999, Albariño \& Díaz-Villanueva 2006), d) historia de vida y emergencias (Hollmann \& Miserendino 2008), y e) patrones latitudinales de diversidad a escala local y regional (Palma \& Figueroa 2008). La mayor parte de los estudios que han incluido Plecoptera han sido realizados en la Patagonia oriental, siendo los del lado occidental todavía escasos (Moorman et al. 2006, Oyanedel et al. 2008), al igual que en el caso de los aéglidos (ver Jara et al. 2006).

En síntesis, el Análisis de Parsimonia de Endemismo permitió hacer un ejercicio comparativo de la composición de la fauna de Plecoptera y Aeglidae e identificar un área de endemismo en la cuenca del río Aysén. La deconstrucción de estos resultados permitió sugerir que para organismos de alta vagilidad (Plecoptera), los acontecimientos históricos tendrían menos influencia que las exigencias ecológicas actuales, en particular de la temperatura. Por el contrario, para el caso de organismos de baja vagilidad (Aeglidae), los fenómenos históricos ocurridos en el Cuaternario parecieran tener mayor relevancia. Estos resultados tendrían particular importancia en el ámbito de la conservación de la biodiversidad dulceacuícola, ya que muestran que los invertebrados de baja vagilidad serían más lábiles al impacto de las actividades antropogénicas debido a sus poblaciones muy localizadas y fragmentadas. Estos resultados debieran ser considerados al momento de tomar decisiones de conservación de la biodiversidad dulceacuícola de la Patagonia chilena. En particular, la cuenca hidrográfica del río Aysén corresponde a una de estas áreas cuya gestión debiera priorizarse.

Es probable que muestreos más exhaustivos permitan determinar otras áreas de endemismo o bien ampliar los rangos distribucionales de las especies. La mayor parte de la información disponible, tanto en colecciones y literatura, corresponde a recolecciones efectuadas cerca de poblados o a la red vial, originando en conjunto una información local incompleta. Recolecciones en sectores de difícil acceso, como la zona de archipiélagos patagónicos, podrían incluir nuevos datos de distribución o establecer otras áreas de endemismo. Finalmente, los resultados obtenidos en este trabajo podrán ser interpretados a la luz de la biogeografía histórica, cuando se reconstruya la filogeografía de algunos Plecoptera y Aeglidae, y se relacionen las divergencias de los taxa con eventos vicariantes que pudieran haber afectado a su área de distribución.

\section{AGRADECIMIENTOS}

Se agradece a Marysol Azócar, Mariela Henríquez, Pablo Pedreros, Gabriela Mancilla, Ricardo Figueroa, Andrés Moraga y Elena Valdovinos, por su colaboración en la obtención y/o análisis de las muestras. Igualmente a los entomólogos Dr. Jorge Artigas y Tomás Cekálovic, por facilitar el material depositado en el Museo Zoológico de la Universidad de Concepción, y a Francisco Riestra, de la Dirección General de Aguas, por su apoyo logístico en terreno. Un reconocimiento especial a nuestro querido amigo Dr. Carlos Jara por introducirnos al mundo de los aéglidos y al Editor Asociado Dr. Rodrigo Ramos Jiliberto, del Departamento de Ciencias Ecológicas de la Facultad de Ciencias de la Universidad de Chile, quien junto a dos revisores anónimos permitieron mejorar substancialmente la versión original del manuscrito. Se agradece al Fondo Nacional de Ciencia y Tecnología (FONDECYT) que financió este estudio a través del proyecto Fondecyt 1070513. Igualmente, se agradece al Proyecto Complementario del Bicentenario (RUE-141) - TWINLATIN y al Proyecto DIUCPatagonia 205.310.043-1sp, por proporcionar información sobre la cuenca del río Baker, y al Proyecto Fondecyt 1050576 que aportó toda la información sobre la cuenca del río Aysén.

\section{LITERATURA CITADA}

ALBARIÑO R \& V DÍAZ-VILLANUEVA (2006) Feeding ecology of two plecopterans in low order Andean-Patagonian streams. International Review of Hydrobiology 91: 122-135.

ALBARIÑO R (1997) Spatial distribution of Plecoptera 
from an Andean-Patagonic lotic environment in Argentina. Revista Brasileira de Biologia 57: 629636.

ALLAN JD (1995) Stream ecology, structure and function of running waters. Chapman \& Hall, London.

ARTHINGTON AH (1990) Latitudinal gradients in insect species richness of Australian lotic systems: A selective review. Tropical Freshwater Biology 2: 179-196.

ATMAR W \& BD PATTERSON (1993) The measure for order and disorder in the distributions of species in fragmented habitat. Oecologia 96: 373-382.

BLACKBURN TM \& KJ GASTON (1996) Spatial patterns in the species richness of birds in the New World. Ecography 19: 369-376.

BOND-BUCKUP G \& L BUCKUP (1994) A Familia Aeglidae (Crustacea, Decapoda Anomura). Arquivos de Zoologia 32: 159-347.

BORNETTE G, C AMOROS, H PIEGAY, T TACHET \& T HEIN (1998) Ecological complexity of wetlands within a river landscape. Biological Conservation 85: 35-45.

BOYERO L (2002) Insect biodiversity in freshwater ecosystems: Is there any latitudinal gradient. Marine and Freshwater Research 53: 753-755.

BROWN JH \& MV LOMOLINO (1998) Biogeography. Sinauer Associates, Sunderland.

CAAMAÑO M (1985) Taxonomía de las ninfas terminales de Plecoptera (Insecta) en tres ritrones preandinos de Riñihue, X Región, Chile. Tesis de grado para optar al título de profesor de Biología y Química. Universidad Austral de Chile, Valdivia.

CAMUS P (2001) Biogeografía marina de Chile continental. Revista Chilena de Historia Natural 74: 587-617.

CARRASCO J, G CASASSA \& A RIVERA (2002) Meteorological and climatological aspects of the Southern Patagonia Icefield. En: Casassa G (ed) The Patagonian Icefields: A unique natural laboratory for environmental and climate change studies: 29-41. Kluwer Academic / Plenum Publishers, New York.

CAVIERES LA, M MIHOC, A MARTICORENA, C MARTICORENA, O MATTHEI \& FA SQUEO (2001) Determinación de áreas prioritarias de conservación: Análisis de parsimonia de endemismos (PAE) en la flora de la IV Región de Coquimbo. En: Squeo FA, G Arancio \& JR Gutiérrez (eds) Libro rojo de la flora nativa y de los sitios prioritarios para su conservación: Región de Coquimbo: 159-170. Ediciones Universidad de La Serena, La Serena, Chile.

CAVIERES LA, MTK ARROYO, P POSADAS, C MARTICORENA, O MATTHEI, R RODRÍGUEZ, FA SQUEO \& G ARANCIO (2002) Identification of priority areas for conservation in an arid zone: Application of parsimony analysis of endemicity in the vascular flora of the Antofagasta region, northern Chile. Biodiversity and Conservation 11: $1303-1311$.

CHAO A (2005) Species richness estimation. En: Balakrishnan N, CB Read \& B Vidakovic (eds) Encyclopedia of statistical sciences: 7909-7916. Wiley, New York.

COLWELL RK (2005) EstimateS. Statistical estimation of species richness and shared species from samples (online). Version 7.5. URL purl.oclc.org/estimates (5.01.10).
COLWELL RK \& DC LEES (2000) The mid-domain effect: geometric constraints on the geography of species richness. Trends in Ecology and Evolution 15: 70-76.

CROZIER L (2004) Warmer winters drive butterfly range expansion by increasing survivorship. Ecology 85: 231-241.

CURRIE D \& V PAQUIN (1987) Large-scale biogeographic patterns of species richness of trees. Nature 329: 326-327.

CURRIE DJ (1991) Energy and large scale patterns of animal and plant species richness. American Naturalist 137: 27-49.

DE POL-HOLZ R, O ULLOA, L DEZILEAU, J KAISER, F LAMY \& D HEBBELN (2006) Melting of the Patagonian Ice Sheet and deglacial perturbations of the nitrogen cycle in the eastern South Pacific. Geophysical Research Letters 33.

DÍAZ-VILLANUEVA V \& R ALBARIÑO (1999) Feeding habit of Notoperla archiplatae (Plecoptera) larvae in a North Patagonia Andean stream, Argentina. Hydrobiologia 412: 43-52.

DI CASTRI F (1968) Esquisse écologique du Chili. En: Delamare CL \& E Rapoport (eds) Biologie de l'Amérique australe: 7-52. Editions Centre National de la Recherche Scientifique, Paris, France.

DONATO M, P POSADAS, DR MIRANDA-ESQUIVEL, EO JAUREGUIZAR \& G CLADERA (2003) Historical biogeography of the Andean region: Evidence from Listroderina (Coleoptera: Curculionidae: Rhytirrhinini) in the context of the South American geobiotic scenario. Biological Journal of the Linnean Society 80 : 339-352.

DOUGLASS DC, BS SINGER, MR KAPLAN, DM MICKLESON \& MW CAFFEE (2006) Cosmogenic nuclide surface exposure dating of boulders on last-glacial and late-glacial moraines, Lago Buenos Aires, Argentina: Interpretative strategies and paleoclimate implications. Quaternary Geochronology 1: 43-58.

DYER B (2000) Systematic review and biogeography of the freshwater fishes of Chile. Estudios Oceanológicos 19: 77-98.

EGUIARTE LE，J LARSON-GUERRA，J NÚNEZFARFÁN, A MARTÍNEZ-PALACIOS, K SANTOS DEL PRADO \& HT ARITA (1999) Diversidad filogenética y conservación: Ejemplos a diferentes escalas. Revista Chilena de Historia Natural 72: 475-492.

ESCALANTE T, D ESPINOSA D \& JJ MORRONE (2003) Using parsimony analysis of endemicity to analyze the distribution of Mexican land mammals. Southwestern Naturalist 48: 563-578.

FIGUEROA R, C VALDOVINOS, E ARAYA \& O PARRA (2003) Macroinvertebrados betónicos como indicadores de calidad de agua de ríos del sur de Chile. Revista Chilena de Historia Natural 76: 275-285.

FISCHER AG (1959) Latitudinal variations in organic diversity. Evolution 14: 64-81.

FOCHETTI R \& J TIERNO DE FIGUEROA (2008) Global diversity of stoneflies (Plecoptera; Insecta) in freshwater. Hydrobiologia 595: 365377.

GAINES SD \& J LUBCHENCO (1982) A unified approach to marine plant herbivore interactions. II. Biogeography. Annual Review in Ecology and Systematics 13: 111-138. 
GARRAFONI ARS, SS NIHEI \& PDC LANA (2006) Distribution patterns 457 of Terebellidae (Annelida: Polychaeta): An application of parsimony analysis of endemicity (PAE). Scientia Marina 70S3: 269-276.

GASTON KJ (2000) Global patterns in biodiversity. Nature 405: 220-227.

GLASSER NF, KN JANSSON, S HARRISON \& J KLEMAN (2008) The glacial geomorphology and Pleistocene history of South America between $38^{\circ} \mathrm{S}$ and $56^{\circ} \mathrm{S}$. Quaternary Science Reviews 27: 365-390.

GOLOBOFF P (1997) NONA versión 2.0 Programa y documentación. New York American Museum of Natural History, USA.

HAMPE A \& RJ PETIT (2005) Conserving biodiversity under climate change: The rear edge matters. Ecology Letters 8: 461-467.

HAUER FR \& VH RESH (2007) Macroinvertebrates. En: Hauer FR \& GA Lamberti (eds) Methods in Stream Ecology: 435-464. Academic Press, San Diego, California.

HAUSDORF B \& C HENNIG (2003) Nestedness of north-west European land snail ranges as a consequence of differential immigration from Pleistocene glacial refuges. Oecologia 135: 102109.

HAWKINS A \& J DINIZ-FILHO (2006) Beyond Rapoport's rule: Evaluating range size patterns of New World birds in a two-dimensional framework. Global Ecology and Biogeography 15: 461-469.

HAWKINS BA, R FIELD, HV CORNELL, DJ CURRIE, JF GUEGAN, et al. (2003) Energy, water, and broad-scale geographic patterns of species richness. Ecology 84: 3105-3117.

HECKMAN C (2003) Encyclopedia of South American aquatic insects: Plecoptera. Kluwer Academic Publishers, Dordrecht, The Netherlands.

HEWITT G (2000) The genetic legacy of the Quaternary ice ages. Nature 405: 907-913.

HILLEBRAND H (2004) On the generality of the latitudinal diversity gradient. American Naturalist 163: 192-211.

HOLLMAN M \& L MISERENDINO (2008) Life history and emergence patterns of stonefly species in mountain streams of the Futaleufú basin, Patagonia (Argentina). International Journal of Limnology 44: 1-10.

ILLIES J (1963) Revision der südamerikanischen Gripopterygidae (Plecoptera). Mitteilungen der Schweizerischen Entomologischen Gesellschaft 36: $145-248$.

ILLIES J (1969) Biogeography and ecology of neotropical freshwater insects especially those from running waters. En: Fittkau EJ, J Illies, H Kling, GH Schwabe \& H Sioli (eds) Biogeography and ecology in South America: 685-708. Dr W Junk Publishers, The Hague, The Netherlands.

INE (2006) Medio Ambiente: Informe anual 2006. Instituto Nacional de Estadísticas, Gobierno de Chile, Santiago.

JACOBSEN D, R SCHUKTZ \& A ENCALADA (1997) Structure and diversity of stream invertebrate assemblages: The influence of temperature with altitude and latitude. Freshwater Biology 38: 247-261.

JARA C, E RUDOLPH \& E GONZÁLEZ (2006) Estado de conocimiento de los crustáceos malacostracos dulceacuícolas de Chile. Gayana 70: $40-49$

JEREZ V (2000) Diversidad y patrones de distribución geográfica de insectos coleópteros en ecosistemas desérticos de la región de Antofagasta, Chile. Revista Chilena de Historia Natural 73: 79-92.

KAPLAN MR, DC DOUGLASS, BS SINGER, RP ACKERT \& MW CAFFEE (2005) Cosmogenic nuclide chronology of pre-last glacial maximum moraines at Lago Buenos Aire, $46^{\circ} \mathrm{S}$, Argentina. Quaternary Research 63: 301-315.

KASPARI M, S O'DONNELL \& JR KERCHER (2000) Energy, density, and constraints to species richness: Ant assemblages along a productivity gradient. American Naturalist 155: 280-293.

KUSCHEL G (1964) Problems concerning an austral region. En: Gressit JL, CH Lindroth, FR Forsberg, A Fleming \& EG Turbott (eds) Pacific basin biogeography: 443-449. Bishop Museum Press, Honolulu, Hawai, USA.

KUSCHEL G (1969) Biogeography and ecology of South American Coleoptera. En: Fittkau J, H Kinge, GH Schawake \& $\mathrm{H}$ Sioli (eds) Biogeography and ecology in South America: 709-722. Dr W Junk Publishers, The Hague, The Netherlands.

LOMOLINO MV (1996) Investigating causality of nestedness of insular communities: Selective immigrations or extinctions? Journal of Biogeography 23: 699-703.

MALMQVIST B (2000) How does wing length relate to distribution patterns of stoneflies (Plecoptera) and mayflies (Ephemeroptera)? Biological Conservation 93: 271-276.

MARQUET PA, M FERNÁNDEZ, SA NAVARRETE \& C VALDOVINOS (2004) Diversity emerging: Towards a deconstruction of biodiversity patterns. En: Lomolino M \& L Heaney (eds) Frontiers of biogeography: New directions in the geography of nature: 191-209. Cambridge University Press, New York.

McLELLAN I, M MERCADO \& S ELLIOTT (2005) A new species of Notoperla (Plecoptera: Gripopterygidae) from Chile. Illiesia 1: 33-39.

McLELLAN I \& P ZWICK (2007) New species of and keys to South American Gripopterygidae (Plecoptera). Illiesia 3: 20-42.

MISERENDINO L (2006) Seasonal and spatial distribution of stoneflies in the Chubut River (Patagonia, Argentina). Hydrobiologia 568: 263274.

MISERENDINO L \& L PIZZOLÓN (2000) Macroinvertebrates of a fluvial system in Patagonia: Altitudinal zonation and functional structure. Archiv für Hydrobiologie 150: 55-83.

MITTERMEIER RA, CG MITTERMEIER, TM BROOKS, JD PILGRIM, WR KONSTANT, GAB DA FONSECA \& C KORMOS (2003) Wilderness and biodiversity conservation. Proceedings of the National Academy of Sciences USA 100: 10309-10313.

MOORMAN C, B ANDERSON, G GUTIÉRREZ, R CHARLIN \& R ROZZI (2006) Watershed conservation and aquatic benthic macroinvertebrate diversity in the Alberto D’Agostini National Park, Tierra del Fuego, Chile. Anales del Instituto de la Patagonia 34: 41-58.

MORRISON LW, MD KORZUKHIN \& SD PORTER (2005) Predicted range expansion of the invasive 
fire ant, Solenopsis invicta, in the eastern United States based on the VEMAP global warming scenario. Diversity and Distributions 11: 199204.

MORRONE JJ (1994) On the identification of areas of endemism. Systematic Biology 43: 438-441.

MORRONE JJ (1996) The biogeographical Andean subregion: A proposal exemplified by Arthropod taxa (Arachnida, Crustacea and Hexapoda). Neotropica 42: 103-114.

MORRONE JJ \& JV CRISCI (1995) Historical biogeography: Introduction to methods. Annual Review of Ecology and Systematics 26: 373-401.

MORRONE JJ, L KATINAS \& JV CRISCI (1997) A cladistic biogeographic analysis of Central Chile. Journal of Comparative Biology 2: 25-42.

MORRONE JJ, S ROIG-JUÑNENT \& JV CRISCI (1994) Cladistic biogeography of terrestrial SubAntartic beetles (Insecta: Coleoptera) from South America. National Geographic Research and Exploration 10: 104-115.

MORRONE JJ, S ROIG-JUÑENT \& G FLORES (2002) Delimitation of biogeographic districts in central Patagonia (South America), based on beetle distributional patterns (Coleoptera: Carabidae and Tenebrionidae). Revista del Museo Argentino de Ciencias Naturales 4: 1-6.

MOYA C, C VALDOVINOS, A MORAGA, F ROMERO, $P$ DEBELS \& A OYANEDEL (2009) Patrones de distribución espacial de ensambles de macroinvertebrados bentónicos de un sistema fluvial andino patagónico. Revista Chilena de Historia Natural 82: 425-442.

MUELLNER AN, K TREMETSBERGER, T STUESSY \& CM BAEZA (2005) Pleistocene refugia and recolonization routes in southern Andes: Insights from Hypochaeris palustris (Asteraceae, Lactuceae). Molecular Ecology 14: 203-212.

MYERS N, RA MITTERMEIER, CG MITTERMEIER, GAB DA FONSECA \& J KENT (2000) Biodiversity hotspots for conservation priorities. Nature 403: 853-858.

NIEMEYER H \& P CERECEDA (1984) Geografía de Chile: Hidrografía. Ed Instituto Geográfico Militar, Santiago, Chile.

NIXON KC (2000) Winclada. Programa y documentación. Publicado por el autor, Ithaca, NY, USA.

NIXON KC (1999) Winclada (BETA) ver. 0.9.9 Published by the author, Ithaca NY.

OYANEDEL A, C VALDOVINOS, M AZÓCAR, C MOYA, G MANCILLA, P PEDREROS \& R FIGUEROA (2008) Patrones de distribución espacial de los macroinvertebrados bentónicos de la cuenca del río Aysén (Patagonia chilena). Gayana 72: 105-121.

OYANEDEL P, C VEGA-RETTER, S SCOTT, LF HINOJOSA \& R RAMOS-JILIBERTO (2008) Finding patterns of distribution for freshwater phytoplankton, zooplankton and fish, by means of parsimony analysis of endemicity. Revista Chilena de Historia Natural 81: 185-203.

PALMA A \& R FIGUEROA (2008) Latitudinal diversity of Plecoptera (Insecta) on local and global Scales. Illiesia 4: 81-90.

PALMA RE, E RIVERA-MILLA, TL YATES, PA MARQUET \& AP MEYNARD (2002) Phylogenetic and biogeographic relationships of the mouse oposum Thylamys (Didelphimorphia, Didelphidae) southern South America.
Molecular Phylogenetics and Evolution 25: 245253.

PATTERSON BD \& W ATMAR (1986) Nested subsets and the structure of insular mammalian faunas and archipelagos. Biological Journal of the Linnean Society 28: 65-82.

PEÑA LE (1966) Ensayo preliminar para dividir Chile en regiones entomofaunísticas, basadas especialmente en la familia Tenebrionidae (Col). Apartado de la Revista Universitaria 51: 210-220.

PÉREZ-LOSADA M, CG JARA, G BOND-BUCKUP \& KA CRANDALL (2002a) Phylogenetic relationships among the species of Aegla (Anomura: Aeglidae) freshwater crabs from Chile. Journal of Crustacean Biology 22: 304-313.

PÉREZ-LOSADA M, CG JARA, G BOND-BUCKUPC \& KA CRANDALL (2002b) Conservation phylogenetics of Chilean freshwater crabs Aegla (Anomura, Aeglidae): Assigning priorities for aquatic habitat protection. Biological Conservation 105: 345-353.

PIZARRO-ARAYA J \& V JEREZ (2004) Distribución geográfica del género Gyriosomus GuérinMéneville, 1834 (Coleoptera: Tenebrionidae): Una aproximación biogeográfica. Revista Chilena de Historia Natural 77: 491-500.

POSADAS P (1996) Distributional patterns of vascular plants in Tierra del Fuego: A study applying parsimony analysis of endemicity (PAE). Biogeographica 72: 161-177.

POSADAS P \& D MIRANDA-ESQUIVEL (1999) El PAE (Parsimony analysis of endemicity) como una herramienta en la evaluación de la biodiversidad. Revista Chilena de Historia Natural 72: 539-546.

RAMOS-JILIBERTO R, JP OYANEDEL, C VEGARETTER, FS VALDOVINOS (2009) Nested structure of plankton communities from Chilean freshwaters. Limnologica 39: 319-324.

RAPOPORT EH (1982) Aerography: Geographical strategies of species. Pergamon Press, New York.

REX MA, CT STUART, RR HESSLER, JA ALLEN, HL SANDERS \& GD WILSON (1993) Global scale latitudinal patterns of species diversity in the deep sea benthos. Nature 365: 636-639.

RINGUELET R (1961) Rasgos fundamentales de la zoogeografía de la Argentina. Physis 22: 151-170.

ROHDE K (1992) Latitudinal gradients in species diversity: The search for the primary cause. Oikos 65: 514-527.

ROHDE K (1999) Latitudinal gradients in species diversity and Rapoport's rule revisited: A review of recent work and what can parasites teach us about the causes of the gradients? Ecography 22: 593-613.

ROIG-JUÑENT S, GE FLORES \& C MATTONI (2003) Consideraciones biogeográficas de la Precordillera (Argentina), con base en artrópodos epígeos. En: Morrone JJ \& J Llorente-Bousquets (eds) Una perspectiva latinoamericana de la biogeografía: 275-288. Las Prensas de Ciencias, Facultad de Ciencias, UNAM, México.

ROY K, D JABLONSKI \& JW VALENTINE (1994) Eastern Pacific molluscan provinces and latitudinal diversity gradient: No evidence for 'Rapoport's rule'. Proceedings of the National Academy of Sciences 91: 8871-8874.

ROY K, D JABLONSKI \& JW VALENTINE (1998) Marine latitudinal diversity gradients: Tests of 
causal hypotheses. Proceedings of the national Academy of Sciences 95: 3699-3702.

ROY K, D JABLONSKI \& JW VALENTINE (2000) Dissecting latitudinal diversity gradients: Functional groups and clades of marine bivalves. Proceedings of the Royal Society B 267: 293-299.

ROSENZWEIG ML \& EA SANDLIN (1997) Species diversity and latitudes: Listening to the area's signal. Oikos 80: 172-176.

RUZZANTE D, SJ WALDE, JC GOSSE, VE CUSSAC, E HABIT, TS ZEMLAK \& EDM ADAMS (2008) Climate control on ancestral population dynamics: Insight from patagonian fish phylogeography. Molecular Ecology 17: 2234-2244.

SAX DF (2001) Latitudinal gradients and geographic ranges of exotic species: Implications for biogeography. Journal of Biogeography 28: 139150.

SHELDON AL (1984) Colonization dynamic of aquatic insects. En: Resh VH \& DM Rossemberg (eds) The ecology of aquatic insects: 401-429. Praeger Publisher, New York.

SINGER BS, RO ACKERT \& H GUILLOU (2004) ${ }^{40} \mathrm{Ar} /$ ${ }^{39} \mathrm{Ar}$ and $\mathrm{K}-\mathrm{Ar}$ chronology of Pleistocene glaciations in Patagonia. Geological Society of America Bulletin 116: 434-450.

SQUEO FA, LA CAVIERES, G ARANCIO, JE NOVOA, O MATTHEI et al. (1998) Biodiversidad de la flora vascular en la Región de Antofagasta, Chile. Revista Chilena de Historia Natural 71: 571-591.

STANFORD J, J WARD, W LISS, C FRISSELL, R WILLIAMS, J LICHATOWICH \& C COUTANT (1996) A general protocol for restoration of regulated rivers. Regulated Rivers: Research and Management 12: 391-413.

STARK B (2007) Anacroneuria marshali (Plecoptera: Perlidae), a new stonefly from Argentina, and two new records from Ecuador. Illiesia 3: 171-173.

STARK B (2008) Diamphipnoa colberti, a new stonefly species from Chile, and the possible female of Diamphipnopsis beschi (Plecoptera: Diamphipnoidae). Illesia 4: 55-58.

STEVENS GC (1989) The latitudinal gradient in geographic range: How so many species coexist in the tropics. The American Naturalist 133: 240256.

SZUMIK C, F CUEZZO, P GOLOBOFF \& A CHALUP (2002) An optimality criterion to determine areas of endemism. Systematic Biology 51: 806-816.

THEISCHINGER G (1991) Plecoptera. En: Theischinger E (ed) The insects of Australia: 311-319. Melbourne University Press, Melbourne, Australia.
TURNER JRG, CM GATEHOUSE \& CA COREY (1987) Does solar energy control organic diversity? Butterflies, moths and the British climate. Oikos 48: 195-205.

VALDOVINOS C (2004) Ecosistemas estuarinos. En: Werlinger C (ed) Biología marina y oceanografía: Conceptos y procesos: 395-414. Trama Impresores S.A., Concepción, Chile.

VALDOVINOS C (2006) Estado de conocimiento de los gastrópodos dulceacuícolas de Chile. Gayana 70: 100-113.

VALDOVINOS C (2008) Invertebrados dulceacuícolas. En: Conama (ed) Biodiversidad de Chile: Patrimonio y desafíos: 202-223. Tercera edición, Ocho Libros Editores, Santiago, Chile.

VALDOVINOS C, S NAVARRETE \& P MARQUET (2003) Mollusk species diversity in the Southeastern Pacific: Why are there more species towards the pole?. Ecography 26: 139144

VERA A \& A CAMOUSSEIGHT (2006) Estado de conocimiento de los plecópteros de Chile. Gayana 70: 57-64.

VINSON MR \& CP HAWKINS (1998) Biodiversity of stream insects: Variation at local, basin and regional scales. Annual Review of Entomology 43: 271-293.

VINSON MR \& CP HAWKINS (2003) Broad-scale geographical patterns in local stream insect genera richness. Ecography 26: 751-767.

WARD JV \& JA STANFORD (1982) Thermal responses in the evolutionary ecology of aquatic insects. Annual Review of Entomology 27: 97-117.

WARD J, K TOCKNER, D ARSCOTT \& C CLARET (2002) Riverine landscape diversity. Freshwater Biology 47: 517-539.

WARD JV (1998) Riverine landscapes: Biodiversity patterns, disturbance regimes, and aquatic conservation. Biological Conservation 83: 269278 .

WILLIG MR, DM KAUFMAN \& RD STEVENS (2003) Latitudinal gradients of biodiversity: Pattern, process, scale, and synthesis. Annual Review of Ecology and Systematics 34: 273-309.

WILLIG MR \& KW SELCER (1989) Bat species density gradients in the New World: A statistical assessment. Journal of Biogeography 16: 189195.

WRIGHT DH, BD PATTERSON, GM MIKKELSON, A CUTLER \& W ATMAR (1998) A comparative analysis for nested subset patterns of species composition. Oecologia 113: 1-20. 
